\title{
INFINITE VOLUME ASYMPTOTICS OF THE GROUND STATE ENERGY IN A SCALED POISSONIAN POTENTIAL
}

\author{
Franz MERKL ${ }^{\text {a }}$, Mario V. WÜTHRICH ${ }^{\mathrm{b}}$ \\ ${ }^{a}$ Universität Bielefeld, Fakultät Mathematik, Postfach 100131, 33501 Bielefeld, Germany \\ ${ }^{\mathrm{b}}$ Winterthur Insurance, Römerstrasse 17, P.O. Box 357, CH-8401 Winterthur, Switzerland
}

Received 31 March 2000, revised 10 July 2001

\begin{abstract}
We investigate the ground state energy of the random Schrödinger operator $-\frac{1}{2} \Delta+\beta(\log t)^{-2 / d} V$ on the box $(-t, t)^{d}$ with Dirichlet boundary conditions. $V$ denotes the Poissonian potential which is obtained by translating a fixed non-negative compactly supported shape function to all the particles of a $d$-dimensional Poissonian point process. The scaling $(\log t)^{-2 / d}$ is chosen to be of critical order, i.e. it is determined by the typical size of the largest hole of the Poissonian cloud in the box $(-t, t)^{d}$. We prove that the ground state energy (properly rescaled) converges to a deterministic limit $I(\beta)$ with probability 1 as $t \rightarrow \infty$. $I(\beta)$ can be expressed by a (deterministic) variational principle. This approach leads to a completely different method to prove the phase transition picture developed in [4]. Further we derive critical exponents in dimensions $d \leqslant 4$ and we investigate the large- $\beta$-behavior, which asymptotically approaches a similar picture as for the unscaled Poissonian potential considered by Sznitman [9]. ๑ 2002 Éditions scientifiques et médicales Elsevier SAS
\end{abstract}

RÉSUMÉ. - Nous examinons l'énergie à l'état de base de l'opérateur de Schrödinger aléatoire $-\frac{1}{2} \Delta+\beta(\log t)^{-2 / d} V$ sur le cube $(-t, t)^{d}$ avec des conditions marginales de Dirichlet. $V$ désigne le potentiel Poissonien obtenu par translation d'une fonction modèle fixe, non-négative et à support compact sur toutes les particules d'un processus de Poisson ponctuel $d$-dimensionnel. L'échelle choisie, $(\log t)^{-2 / d}$, est d'ordre critique, c'est-à-dire qu'elle est déterminée par la taille typique du plus grand espace vide dans le nuage Poissonien sur le cube $(-t, t)^{d}$. Nous démontrons que l'énergie à l'état de base (convenablement rééchelonnée) converge vers une limite déterministe $I(\beta)$ avec probabilité 1 larsque $t \rightarrow \infty$. $I(\beta)$ peut être exprimée à l'aide d'un principe variationnel (déterministe). Cette approche conduit à une méthode complètement différente pour prouver l'image de transition de phase développée dans [4]. De plus nous obtenons des exposants critiques pour les dimensions $d \leqslant 4$ et nous examinons le comportement pour les grandes valeurs de $\beta$, qui approche asymptotiquement une image semblable à celle du potentiel Poissonien non-échelonné considéré par Sznitman [9]. ๑ 2002 Éditions scientifiques et médicales Elsevier SAS

E-mail addresses: merkl@mathematik.uni.bielefeld.de (F. Merkl), mario.wuethrich@winterthur.ch (M.V. Wüthrich). 


\section{Introduction and results}

In this article, we consider the infinite volume limit of the ground state energy (principal Dirichlet eigenvalue) for a non-relativistic quantum particle in a scaled Poissonian potential. The motivation for this study is to develop a better understanding of the corresponding (random) variational problem and its phase transition picture proven in [4]. Related random variational problems naturally arise in several questions of disordered media, e.g. in the study of the path behavior of Brownian motion in a Poissonian potential: the Poissonian potential plays the role of an absorption rate, and one tries to determine where the surviving Brownian particles settle down (see [9], Section 6.1). In the main body of this article we first derive a (deterministic) variational principle for the infinite volume limit of the (rescaled) ground state energy of the random Schrödinger operator. In the second part we analyze this variational problem and derive the phase transition picture.

We start with the definition of the scaled (random) potential: it is obtained by translating a fixed shape function $W$ to all the points of a Poissonian cloud (of constant intensity $v=1)$. Let $\mathbb{P}$ stand for the canonical law of the Poissonian point process $\omega=\sum_{i} \delta_{x_{i}} \in \Omega$ (where $\Omega$ is the set of all simple pure locally finite point measures on $\mathbb{R}^{d}$ ). The scaled Poissonian potential with scaling function $\varphi: \mathbb{R}_{+} \rightarrow \mathbb{R}_{+}$is then defined as follows: for $x \in \mathbb{R}^{d}, \beta>0, t>0, \omega \in \Omega$ we set

$$
\begin{aligned}
V_{\beta, t}^{\varphi}(x, \omega) \stackrel{\text { def }}{=} \frac{\beta}{\varphi(t)^{2}} V(x, \omega) & \stackrel{\text { def }}{=} \frac{\beta}{\varphi(t)^{2}} \sum_{i} W\left(x-x_{i}\right) \\
& =\frac{\beta}{\varphi(t)^{2}} \int W(x-y) \omega(\mathrm{d} y),
\end{aligned}
$$

where we assume that the shape function $W \geqslant 0$ is measurable, bounded, compactly supported, and $\int W(x) \mathrm{d} x=1$. When it causes no ambiguity, we shall omit to write superscripts $\varphi$.

For any non-negative potential $V$ and $\phi \in H_{0}^{1,2}\left(\mathbb{R}^{d}\right)$ we introduce the quadratic form

$$
\mathcal{E}_{V}(\phi) \stackrel{\text { def }}{=} \frac{1}{2}\|\nabla \phi\|_{2}^{2}+\int_{\mathbb{R}^{d}} V \phi^{2} \mathrm{~d} x .
$$

Then the ground state energy on a non-empty open set $U \subset \mathbb{R}^{d}$ of the potential $V_{\beta, t}^{\varphi}$ is defined as follows (see also Sznitman [9], (3.1.2))

$$
\begin{aligned}
\lambda_{V_{\beta, t}^{\varphi}}(U) & \stackrel{\text { def }}{=} \inf \left\{\mathcal{E}_{V_{\beta, t}^{\varphi}}(\phi): \phi \in H_{0}^{1,2}(U),\|\phi\|_{2}=1\right\} \\
& =\text { principal Dirichlet eigenvalue of }-\frac{1}{2} \Delta+V_{\beta, t}^{\varphi} \text { in } U,
\end{aligned}
$$

where $H_{0}^{1,2}(U)$ is the Sobolev space with generalized Dirichlet boundary conditions on $U$.

Let $\Phi$ denote the set of all test functions $\phi \in H_{0}^{1,2}\left(\mathbb{R}^{d}\right)$ which are continuous, compactly supported, and normalized: $\|\phi\|_{2}=1$. For $\phi \in \Phi$ we define the logarithmic 
moment generating function of the Poisson process:

$$
\Lambda_{\phi}(\sigma) \stackrel{\text { def }}{=} \log \mathbb{E}\left[\exp \left\{\sigma \int_{\mathbb{R}^{d}} \phi^{2} \mathrm{~d} \omega\right\}\right]=\int_{\mathbb{R}^{d}}\left(\mathrm{e}^{\sigma \phi^{2}}-1\right) \mathrm{d} x, \quad \sigma \in \mathbb{R},
$$

and its one-dimensional Fenchel-Legendre transform, $\mu \in \mathbb{R}$,

$$
\Lambda_{\phi}^{*}(\mu) \stackrel{\text { def }}{=} \sup _{\sigma \in \mathbb{R}}\left(\sigma \mu-\Lambda_{\phi}(\sigma)\right) .
$$

We introduce the following function: For $\beta \geqslant 0$,

$$
I(\beta) \stackrel{\text { def }}{=} \inf \left\{\frac{1}{2}\|\nabla \phi\|_{2}^{2}+\beta \mu: \phi \in \Phi, \mu \in(0,1), \Lambda_{\phi}^{*}(\mu) \leqslant d\right\} .
$$

Our first main result is the following variational principle:

THEOREM 0.1. - For $d \geqslant 1, \beta>0$ and $\varphi(t)=(\log t)^{1 / d}$ the following holds:

$$
\mathbb{P} \text {-a.s. } \quad \lim _{t \rightarrow \infty}(\log t)^{2 / d} \lambda_{V_{\beta, t}^{\varphi}}\left((-t, t)^{d}\right)=I(\beta) .
$$

The new result here is that the above limit exists $\mathbb{P}$-a.s. and that it is $\mathbb{P}$-a.s. equal to the deterministic number coming from the variational principle (0.6).

A statement similar to Theorem 0.1 is valid for general $W \geqslant 0$ (measurable, compactly supported, bounded, with positive $L^{1}$ norm) and general Poisson intensity $v>0$. We only treat the case $\int W \mathrm{~d} x=1$ and $v=1$, since this covers already the whole flavor of the problem and the general case can be recovered by a simple scaling argument.

We compare Theorem 0.1 with the earlier results without scaling of the potential (see Sznitman [8], and [9], Theorem 4.4.6): remarkably in the unscaled picture, the ground state energy asymptotically does not depend on the choice of the shape function $\beta W$, as long as this function does not vanish identically:

$$
\mathbb{P} \text {-a.s. } \quad \lim _{t \rightarrow \infty}(\log t)^{2 / d} \lambda_{\beta V}\left((-t, t)^{d}\right)=c(d, 1),
$$

where $c(d, 1)$ is the principal Dirichlet eigenvalue of $-\frac{1}{2} \Delta$ on a $d$-dimensional ball of volume $d$. The constant $c(d, 1)$ was introduced by Sznitman [9], formula (4.4.20). The known result (0.8) may be contrasted with formula (0.7): in the scaled picture, there is one relevant parameter of the shape function $\beta W$, namely its $L^{1}$-norm $\beta$, while all other details of the potential still remain irrelevant for the leading term asymptotics of the ground state energy.

In the unscaled picture, one sees by the "method of enlargement of obstacles" (Sznitman [9]), that the principal Dirichlet eigenfunctions are very close to 0 over regions with already a rather low concentration of Poissonian points (obstacles): one can subtract such domains from the box $(-t, t)^{d}$ and the ground state energy essentially remains unchanged on the new smaller domain. Hence it suffices to consider the subdomains of $(-t, t)^{d}$ which are (almost) not to be affected by the Poissonian point process. 
Quite differently, in the scaled picture, we search for optimally shaped test functions supported in certain "optimal" large deviations of the Poissonian clouds; these "optimal" regions typically contain a considerable number of Poissonian points. It turns out that a smoothing procedure on a length scale much larger than the size of the support of $W$ does not considerably change the ground state energy; almost all details of the shape function $\beta W$ get essentially lost during this smoothing (convolution) procedure, except the $L^{1}$-norm $\beta=\|\beta W\|_{1}=\mathbb{E}[\beta V(0, \omega)]$, which is invariant with respect to convolution with non-negative normalized $L^{1}$-functions.

In the limit $\beta \rightarrow \infty$, we recover an analogous asymptotic behavior as in the unscaled picture:

THEOREM 0.2 (Large- $\beta$-behavior). - Let $d \geqslant 1$. The function I is concave, nonnegative, strictly monotonically increasing on $[0, \infty)$ with

$$
\lim _{\beta \rightarrow \infty} I(\beta)=c(d, 1) .
$$

More quantitative estimates for the speed of convergence in (0.9) as $\beta \rightarrow \infty$ are provided in Lemmas 3.5 and 3.6 below.

THEOREM 0.3 (Critical exponents in low dimensions). - Let $d \leqslant 3$. There exists a constant $C_{1}(d)>0$ and for every $b_{1}>0$ there exists a constant $C_{2}\left(d, b_{1}\right)>0$ such that for every $\beta \in\left(0, b_{1}\right)$

$$
\beta-C_{1} \beta^{4 /(4-d)} \leqslant I(\beta) \leqslant \beta-C_{2} \beta^{4 /(4-d)} .
$$

The new piece here is the lower bound, while the upper bound is contained in [4], Lemma 3.4. One should compare the above statement with the following theorem:

THEOREM 0.4 (Phase transition in high dimensions). - Let $d \geqslant 4$. There exists $a$ constant $\beta_{c}(d)>0$ such that

$$
\begin{aligned}
& \text { for all } \beta \leqslant \beta_{c}(d) \quad I(\beta)=\beta, \\
& \text { for all } \beta>\beta_{c}(d) \quad I(\beta)<\beta,
\end{aligned}
$$

where the following bounds hold for $\beta_{c}(d)$ :

$$
\begin{aligned}
& \text { For } d=4 \quad \beta_{c}(4)=\frac{\pi}{\sqrt{3}} . \\
& \text { For } d \geqslant 5 \quad d^{1-4 / d}(d-2) 2^{4 / d-3} \pi^{1+1 / d} \Gamma\left(\frac{1+d}{2}\right)^{-2 / d} \leqslant \beta_{c}(d)<c(d, 1) .
\end{aligned}
$$

I.e., in high dimensions we observe a phase transition of the ground state energy on the scale $\varphi(t)=(\log t)^{1 / d}$. For small $\beta$ we can choose as test function a normalized approximation of the constant and we obtain already the correct leading order of the asymptotic behavior of the ground state energy. For large $\beta$ the situation is completely different, namely it is more favorable to localize the test function in regions where the number of Poissonian particles is below its average value. In low dimensions this picture does not hold true, namely for any positive value of $\beta$ one should localize the 
test function, i.e. we do not see a phase transition on this scale. (Some parts of these pictures have already been developed in [4].)

THEOREM 0.5 (Critical exponent in 4 dimensions). - Let $d=4$. There exist constants $C_{3}, C_{4}, C_{5}>0$ and $b_{2}>\beta_{c}(4)$ such that for every $\beta \in\left(\beta_{c}(4), b_{2}\right)$

$$
\beta-C_{3}\left(\beta-\beta_{c}(4)\right)^{2} \leqslant I(\beta) \leqslant \beta-C_{4} \frac{\left(\beta-\beta_{c}(4)\right)^{2}}{\left|\log \left[C_{5}\left(\beta-\beta_{c}(4)\right)\right]\right|} .
$$

We remark that there is numerical evidence that the above picture does not hold true for $d \geqslant 5$, i.e. we expect in large dimensions that the derivative $I^{\prime}(\beta)$ should have a jump singularity at $\beta=\beta_{c}(d)$. This also corresponds to the picture emphasized in [4].

The next theorem holds for all dimensions, but is mainly interesting for $d \leqslant 3$ : It implies that we are considering the correct scaling, and formally we may write $\beta_{c}(d)=0$ for $d \leqslant 3$ : (We write $a(t) \ll b(t)$ for $a(t) / b(t) \stackrel{t \rightarrow \infty}{\longrightarrow} 0$.)

THEOREM 0.6 (Absence of a phase transition on other scalings). - For $d \geqslant 1, \beta>0$ and $(\log t)^{1 / d} \ll \varphi(t) \ll t$ the following holds:

$$
\mathbb{P} \text {-a.s. } \quad \lim _{t \rightarrow \infty} \varphi(t)^{2} \lambda_{V_{\beta, t}^{\varphi}}\left((-t, t)^{d}\right)=\beta .
$$

Small scalings are treated by the following corollary of Theorem 0.2 :

COROllary 0.7. - For $d \geqslant 1, \beta>0$ and $\varphi(t) \ll(\log t)^{1 / d}$ the following holds:

$$
\text { P-a.s. } \quad \lim _{t \rightarrow \infty}(\log t)^{2 / d} \lambda_{V_{\beta, t}^{\varphi}}\left((-t, t)^{d}\right)=c(d, 1) .
$$

Let us explain how this article is organized: In Section 1 we do all the preparatory work. We introduce some further definitions and we recall some already known results including the upper bound in Theorem 0.1 .

In Section 2 we provide the lower bounds of Theorems 0.1 and 0.6. In a first step we show that if we allow a small error of order $\varphi(t)^{-2}$ we can restrict the infimum in (0.3) (for $U=(-t, t)^{d}$ ) to finitely many smooth test functions which live on balls with radius of order $\varphi(t)$. The main ingredients here are a cutoff (or localization) procedure for eigenfunctions and the compactness property of convolution operators. In a second step then, we derive for all of these finitely many compactly supported smooth test functions a large deviation result estimating the potential term in (0.2). Putting these estimates together yields the lower bounds of Theorems 0.1 and 0.6.

In Section 3 we prove Theorems 0.2-0.5. The main body in the analysis of the variational problem (0.6) is to calculate good upper and lower bounds on the logarithmic moment generating function in order to control the Fenchel-Legendre transform. This is done using scaling arguments, Taylor expansions and Sobolev inequalities. Using these estimates we prove the lower bounds in Theorems 0.3-0.5. (The upper bounds of Theorems 0.3 and 0.4 have already been proven in [4].)

The idea behind the proof of the upper bound in Theorem 0.5 is the following: The Sobolev inequality (3.15) we choose to prove the lower bound turns into an equality (for $d=4$ ) if we choose an appropriate test function. This test function is not in $H_{0}^{1,2}\left(\mathbb{R}^{4}\right)$, 
so we have to take a compactly supported approximation to evaluate (0.2) which then gives the desired leading order. We remark that many of these Sobolev inequalities, we are using here, have already been very helpful in the analysis of a variational problem studied by van den Berg, Bolthausen and den Hollander [2].

The upper bound in Theorem 0.2 follows already from the simple fact $I(\beta) \leqslant c(d, 1)$. However, we give a finer upper and lower estimate for $I(\beta)$ in the large- $\beta$-regime, using the asymptotics of the ground state energy in a deterministic square well potential. This asymptotics is well known to physicists, but unfortunately we were not able to provide a rigoros reference; this is why we describe the argument in Appendix B. The upper estimate for $I(\beta)$ in the large- $\beta$-regime improves a previously known bound (see Theorem 0.1 and Lemma 3.5 in [4]).

Finally in Appendix A we prove the upper bound of Theorem 0.6 using as test function (to evaluate $(0.2)$ ) a normalized approximation to a constant function on $(-t, t)^{d}$. Further we sketch the proof of Corollary 0.7 .

\section{Preliminaries}

We start with the following definitions: For $t>0$, we define

$$
\mathcal{T}_{t} \stackrel{\text { def }}{=}(-t, t)^{d},
$$

$W_{\infty} \stackrel{\text { def }}{=} \sup _{x \in \mathbb{R}^{d}} W(x)$, and $a$ denotes the minimal radius such that supp $W \subseteq \bar{B}_{a}(0)$, where $B_{a}(0)$ is the open ball with center 0 and radius $a$. For $\beta>0$ and $m \in(0,1)$ we define the following functions:

$$
\begin{aligned}
\tilde{I}_{1}(\beta, m) & \stackrel{\text { def }}{=} \inf \left\{\frac{1}{2}\|\nabla \phi\|_{2}^{2}+\beta m \mu: \phi \in \Phi, \mu \in(0,1), \Lambda_{\phi}^{*}(\mu) \leqslant \frac{d}{m}\right\}, \\
I_{1}(\beta) & \stackrel{\text { def }}{=} \lim _{m \uparrow 1} \tilde{I}_{1}(\beta, m), \\
I_{2}(\beta) & \stackrel{\text { def }}{=} \inf \left\{\frac{1}{2}\|\nabla \phi\|_{2}^{2}+\beta \mu: \phi \in \Phi, \mu \in(0,1), \Lambda_{\phi}^{*}(\mu)<d\right\} .
\end{aligned}
$$

The limit on the right-hand side of (1.3) is well-defined since its argument is monotonically increasing in $m: I_{1}(\beta) \geqslant \tilde{I}_{1}(\beta, m) \rightarrow I_{1}(\beta)$ as $m \uparrow 1$.

The upper bound in (0.7) is a consequence of the following considerations: If we choose $\varphi(t)=(\log t)^{1 / d}$ then we have seen in Lemma 3.2 of [4] that for all $\phi \in \Phi$ and $\mu \in(0,1)$ with $\Lambda_{\phi}^{*}(\mu)<d$ we have $\mathbb{P}$-a.s. $\lim \sup _{t \rightarrow \infty}(\log t)^{2 / d} \lambda_{V_{\beta, t}}\left(\mathcal{T}_{t}\right) \leqslant \frac{1}{2}\|\nabla \phi\|_{2}^{2}+$ $\beta \mu$. Hence we obtain $\mathbb{P}$-a.s.

$$
\limsup _{t \rightarrow \infty}(\log t)^{2 / d} \lambda_{V_{\beta, t}}\left(\mathcal{T}_{t}\right) \leqslant I_{2}(\beta) \leqslant \frac{1}{2}\|\nabla \phi\|_{2}^{2}+\beta \mu .
$$

Repeating the argument of Lemma 3.3 in [4], we see that we can choose two sequences $\mu_{n} \in(0,1)$ and $\phi_{n} \in \Phi$ such that $\mu_{n} \uparrow 1($ as $n \rightarrow \infty)$ and $\Lambda_{\phi_{n}}^{*}\left(\mu_{n}\right)<d$ for all $n \geqslant 1$ (see 
formulas (3.28)-(3.29) of [4]) with

$$
\lim _{n \rightarrow \infty} \frac{1}{2}\left\|\nabla \phi_{n}\right\|_{2}^{2}+\beta \mu_{n}=\beta
$$

Henceforth we obtain

$$
\text { P-a.s. } \quad \limsup _{t \rightarrow \infty}(\log t)^{2 / d} \lambda_{V_{\beta, t}}\left(\mathcal{T}_{t}\right) \leqslant I_{2}(\beta) \leqslant \beta \text {. }
$$

Furthermore we have for all $\beta>0$ and $m \in(0,1)$

$$
\tilde{I}_{1}(\beta, m) \leqslant I_{1}(\beta) \leqslant I(\beta) \leqslant I_{2}(\beta) \leqslant \beta
$$

LEMMA 1.1. - There exists $c_{6}>0$ such that for all $\beta>0, \eta>0$ and $m \in(1 / 2,1)$ with

$$
1-m \leqslant c_{6} \eta \beta^{-1}
$$

the following holds:

$$
I_{2}(\beta) \leqslant \tilde{I}_{1}(\beta, m)+\eta \text {. }
$$

Proof. - The function $m \mapsto m^{-4 / d}+\left[\left(1-m^{1-4 / d}\right) \vee 0\right]$, with $\frac{1}{2} \leqslant m \leqslant 1$, has value 1 for $m=1$ and is Lipschitz continuous. Let $1 / c_{6}$ be a strict upper bound for its Lipschitz constant. Then for all $\beta>0, \eta>0$ and $1 / 2<m<1$ which fulfill assumption (1.9) we have

$$
\left(m^{-4 / d}-1\right)+\left[\left(1-m^{1-4 / d}\right) \vee 0\right]<\eta \beta^{-1} .
$$

From now on we fix such a triple $\beta, \eta, m$, hence we can choose $\varepsilon>0$ so small that the following holds:

$$
\left(m^{-4 / d}-1\right) \beta+m^{-4 / d} \varepsilon+\left[\left(1-m^{1-4 / d}\right) \vee 0\right] \beta \leqslant \eta .
$$

By the definition of $\tilde{I}_{1}(\beta, m)$ there are $\phi \in \Phi$ and $\mu \in(0,1)$ with $\Lambda_{\phi}^{*}(\mu) \leqslant \frac{d}{m}$ and

$$
\frac{1}{2}\|\nabla \phi\|_{2}^{2}+\beta m \mu \leqslant \tilde{I}_{1}(\beta, m)+\varepsilon .
$$

For $r>0$ we scale $\phi$ by

$$
\phi_{r}(x) \stackrel{\text { def }}{=} r^{-d / 2} \phi(x / r) .
$$

$\phi_{r}$ scales as follows

$$
\begin{array}{rll}
\left\|\phi_{r}\right\|_{2}^{2}=1 & \text { and } & \left\|\nabla \phi_{r}\right\|_{2}^{2}=r^{-2}\|\nabla \phi\|_{2}^{2}, \\
\Lambda_{\phi_{r}}(\sigma)=r^{d} \Lambda_{\phi}\left(r^{-d} \sigma\right) & \text { and } \quad \Lambda_{\phi_{r}}^{*}(\mu)=r^{d} \Lambda_{\phi}^{*}(\mu) .
\end{array}
$$

For $r(m) \stackrel{\text { def }}{=} m^{2 / d}$ we have $\Lambda_{\phi_{r}}^{*}(\mu)=r^{d} \Lambda_{\phi}^{*}(\mu) \leqslant \frac{r^{d} d}{m}=m d<d$, and 


$$
\begin{aligned}
I_{2}(\beta) & \leqslant \frac{1}{2}\left\|\nabla \phi_{r}\right\|_{2}^{2}+\beta \mu=\frac{1}{2 r^{2}}\|\nabla \phi\|_{2}^{2}+\beta \mu \\
& =m^{-4 / d}\left(\frac{1}{2}\|\nabla \phi\|_{2}^{2}+\beta m \mu\right)+\left(1-m^{1-4 / d}\right) \beta \mu \\
& \leqslant m^{-4 / d}\left(\tilde{I}_{1}(\beta, m)+\varepsilon\right)+\left(1-m^{1-4 / d}\right) \beta \mu \\
& \leqslant \tilde{I}_{1}(\beta, m)+\left(m^{-4 / d}-1\right) \beta+m^{-4 / d} \varepsilon+\left[\left(1-m^{1-4 / d}\right) \vee 0\right] \beta \\
& \leqslant \tilde{I}_{1}(\beta, m)+\eta .
\end{aligned}
$$

We used (1.13), (1.8), and (1.12) in the last three steps. This proves (1.10) and therefore Lemma 1.1.

The upper bound in (0.7) is a consequence of (1.7) and the following corollary:

Corollary 1.2. $-I_{1}(\beta)=I(\beta)=I_{2}(\beta)$.

Proof. - This is a trivial consequence of the bounds (1.8) and the previous Lemma 1.1.

\section{Proof of the lower bounds in (0.7) and (0.16)}

We assume that $\varphi$ is a fixed positive scaling function with $\varphi(t) \rightarrow \infty$ as $t \rightarrow \infty$. We suppress superscripts $\varphi$ when no ambiguity arises.

\subsection{Localization, compactness argument, and large deviations}

Our first step consists of a localization argument: To evaluate $(0.3)$ on $U=\mathcal{T}_{t}$ it suffices to consider test functions supported in balls with radius $R \varphi(t)$, if we allow a small error $\varphi(t)^{-2} \eta$ (see Lemma 2.1). In a second step (Lemma 2.2) we allow another small error $\varphi(t)^{-2} \eta$ to smoothen the test functions. In a third step (Lemma 2.3) we prove that we can restrict ourselves to finitely many smooth test functions if we allow an additional small error of $\varphi(t)^{-2} \eta$. Finally in Lemma 2.5 we give for every of these finitely many smooth test functions a large deviation result estimating the potential term in $(0.2)$.

For $t>0, R>0$ and $y \in d^{-1 / 2} R \varphi(t) \mathbb{Z}^{d}$ we define $B_{R, t, y}^{\varphi} \stackrel{\text { def }}{=} B_{R \varphi(t)}(y)$, and we set $Y_{R, t}^{\varphi} \stackrel{\text { def }}{=}\left\{y \in d^{-1 / 2} R \varphi(t) \mathbb{Z}^{d}: B_{R, t, y} \cap \mathcal{T}_{t} \neq \emptyset\right\}$. Then $\left(B_{R, t, y}\right)_{y \in Y_{R, t}}$ is an open covering of $\mathcal{T}_{t}$.

LEMMA 2.1. - There exists $c_{7}(d)>0$ such that for $\beta>0, \eta>0, R \stackrel{\text { def }}{=}\left(c_{7} / \eta\right)^{1 / 2} \vee 1$ and $t>0$

$$
\lambda_{V_{\beta, t}}\left(\mathcal{T}_{t}\right) \geqslant \min _{y \in Y_{R, t}} \inf _{\substack{\phi \in H_{0}^{1,2}\left(B_{R, t, y}\right) \\\|\phi\|_{2}=1}} \mathcal{E}_{V_{\beta, t}}(\phi)-\varphi(t)^{-2} \eta .
$$

Proof. - Let $c_{8}(d)<\infty$ denote an upper bound for the number of balls $B_{R, t, y}$, $y \in Y_{R, t}$, that intersect $B_{R, t, 0}$ (this number does neither depend on $R>0$ nor on $t>0$ ). We use a partition of unity: Choose $\chi \in C_{c}^{\infty}\left(B_{1}(0)\right)$ a fixed non-negative function with 
$\sum_{j \in d^{-1 / 2} \mathbb{Z}^{d}} \chi(x-j)^{2}=1$ for all $x \in \mathbb{R}^{d}$, and define $c_{7}(d) \stackrel{\text { def }}{=} \frac{c_{8}}{2}\left\||\nabla \chi|^{2}\right\|_{\infty}$. For $\eta>0$, $R=\left(c_{7} / \eta\right)^{1 / 2} \vee 1$ and $t>0$ we define a partition of unity over $\mathcal{T}_{t}$,

$$
1=\sum_{y \in Y_{R, t}} \chi_{R, t, y}^{2} \text { on } \mathcal{T}_{t}
$$

where $\chi_{R, t, y}(x) \stackrel{\text { def }}{=} \chi\left(\frac{x-y}{R \varphi(t)}\right)$ are compactly supported in $B_{R, t, y}$. Next we denote by $\psi \in H_{0}^{1,2}\left(\mathcal{T}_{t}\right)$ a principal Dirichlet eigenfunction of the Schrödinger operator $-\frac{1}{2} \Delta+V_{\beta, t}$ in $\mathcal{T}_{t}$. With the help of the partition of unity we split this eigenfunction into pieces:

$$
\psi^{2}=\sum_{y \in Y_{R, t}}\left(\chi_{R, t, y} \psi\right)^{2}
$$

The definition of $R$ implies the following upper bound on the derivatives of $\chi_{R, t, y}$ :

$$
\frac{1}{2}\left\|\sum_{y \in Y_{R, t}}\left|\nabla \chi_{R, t, y}\right|^{2}\right\|_{\infty} \leqslant \frac{c_{8}}{2}\left\|\left|\nabla \chi_{R, t, 0}\right|^{2}\right\|_{\infty} \leqslant \eta \varphi(t)^{-2} .
$$

We claim for all $\lambda \in \mathbb{R}$ : If

$$
\mathcal{E}_{V_{\beta, t}}\left(\chi_{R, t, y} \psi\right) \geqslant \lambda\left\|\chi_{R, t, y} \psi\right\|_{2}^{2}, \quad \text { for all } y \in Y_{R, t},
$$

then

$$
\mathcal{E}_{V_{\beta, t}}(\psi) \geqslant\left(\lambda-\eta \varphi(t)^{-2}\right)\|\psi\|_{2}^{2} .
$$

Lemma 2.1 is a consequence of this claim: To see this, we observe that the left-hand side in (2.6) equals $\lambda_{V_{\beta, t}}\left(\mathcal{T}_{t}\right)\|\psi\|_{2}^{2}$. Choose $\lambda>\lambda_{V_{\beta, t}}\left(\mathcal{T}_{t}\right)+\eta \varphi(t)^{-2}$; then (2.6) cannot be true. Therefore, we conclude that (2.5) has to fail for at least one $y \in Y_{R, t}$ for this choice of $\lambda$. We set $\phi_{y} \stackrel{\text { def }}{=}\left(\chi_{R, t, y} \psi\right) /\left\|\chi_{R, t, y} \psi\right\|_{2}$ for such a $y \in Y_{R, t}$ (note that $\left\|\chi_{R, t, y} \psi\right\|_{2}$ cannot vanish for this choice of $y$ ). We obtain $\mathcal{E}_{V_{\beta, t}}\left(\phi_{y}\right)<\lambda$. Henceforth, $\min _{y \in Y_{R, t}^{\prime}} \mathcal{E}_{V_{\beta, t}}\left(\phi_{y}\right)<\lambda$ for all $\lambda>\lambda_{V_{\beta, t}}\left(\mathcal{T}_{t}\right)+\eta \varphi(t)^{-2}$, where $Y_{R, t}^{\prime} \stackrel{\text { def }}{=}\left\{y \in Y_{R, t}:\left\|\chi_{R, t, y} \psi\right\|_{2}>0\right\}$. But this implies claim (2.1) of Lemma 2.1.

There remains to prove that (2.5) implies (2.6). We sum (2.5) over all $y \in Y_{R, t}$ and use (2.3) to obtain

$$
\frac{1}{2}\left\|\sum_{y \in Y_{R, t}}\left|\nabla\left(\chi_{R, t, y} \psi\right)\right|^{2}\right\|_{1}+\int V_{\beta, t} \psi^{2} \mathrm{~d} x=\sum_{y \in Y_{R, t}} \mathcal{E}_{V_{\beta, t}}\left(\chi_{R, t, y} \psi\right) \geqslant \lambda\|\psi\|_{2}^{2} .
$$

To estimate the gradient term in (2.7), we take the derivative of formula (2.2): $2 \sum_{y \in Y_{R, t}} \chi_{R, t, y} \nabla \chi_{R, t, y}=0$. Hence using (2.4) we have

$$
\begin{aligned}
& \frac{1}{2}\left\|\sum_{y \in Y_{R, t}}\left|\nabla\left(\chi_{R, t, y} \psi\right)\right|^{2}\right\|_{1} \\
& \quad=\frac{1}{2}\|\nabla \psi\|_{2}^{2}+\left\langle\sum_{y \in Y_{R, t}} \chi_{R, t, y} \nabla \chi_{R, t, y}, \psi \nabla \psi\right\rangle+\frac{1}{2}\left\|\sum_{y \in Y_{R, t}}\left|\nabla \chi_{R, t, y}\right|^{2} \psi^{2}\right\|_{1}
\end{aligned}
$$




$$
\leqslant \frac{1}{2}\|\nabla \psi\|_{2}^{2}+\eta \varphi(t)^{-2}\|\psi\|_{2}^{2} \text {. }
$$

Collecting (2.7) and (2.8) yields (2.6). This finishes the proof of Lemma 2.1.

The next step consists of a smoothening and scaling argument: We take the convolution of test functions with an approximation $\delta_{\varepsilon}$ of Dirac's $\delta$ : Let $\delta_{1} \in C_{c}^{\infty}\left(B_{1}(0)\right)$ be fixed non-negative with $\left\|\delta_{1}\right\|_{1}=1$. Set $\delta_{\varepsilon}(x) \stackrel{\text { def }}{=} \varepsilon^{-d} \delta(x / \varepsilon)$. Let $*$ denote the convolution operator. We set for $R>0$ and $N>0$ :

$$
\Phi_{R, N} \stackrel{\text { def }}{=}\left\{\phi \in H_{0}^{1,2}\left(B_{R}(0)\right):\|\phi\|_{2}=1,\|\nabla \phi\|_{2} \leqslant \sqrt{N}\right\} .
$$

For $t>0, y \in \mathbb{R}^{d}$, we define the scaling operator $S_{t, y}^{\varphi}$ by

$$
\left(S_{t, y}^{\varphi} \phi\right)(x) \stackrel{\text { def }}{=} \varphi(t)^{-d / 2} \phi\left(\varphi(t)^{-1}(x-y)\right) .
$$

This operator maps $H_{0}^{1,2}\left(B_{R}(0)\right)$ onto $H_{0}^{1,2}\left(B_{R, t, y}\right)$; it fulfills

$$
\left\|S_{t, y} \phi\right\|_{2}=\|\phi\|_{2} \quad \text { and } \quad\left\|\nabla\left(S_{t, y} \phi\right)\right\|_{2}=\varphi(t)^{-1}\|\nabla \phi\|_{2} .
$$

We choose a truncation level $M>0$ (to be specified later) and define $V^{M} \stackrel{\text { def }}{=} V \wedge M$. Further we introduce

$$
V_{\beta, t}^{M, \varphi} \stackrel{\text { def }}{=} \beta \varphi(t)^{-2} V^{M} .
$$

For every test function $\phi$ we get the simple but useful inequality

$$
\mathcal{E}_{V_{\beta, t}}(\phi) \geqslant \mathcal{E}_{V_{\beta, t}^{M}}(\phi)
$$

LEMMA 2.2. - There exist $c_{9}(d)>0, c_{10}(d)>0$ such that for all $\beta>0, \eta>0$, $M>0, R=\left(c_{7} / \eta\right)^{1 / 2} \vee 1, N \stackrel{\text { def }}{=} 2 \beta M+c_{10}, \varepsilon \in\left(0, \eta\left(2 \beta M c_{9} N^{1 / 2}\right)^{-1}\right]$, and for all $t>0$ we have:

$$
\lambda_{V_{\beta, t}}\left(\mathcal{T}_{t}\right) \geqslant \min _{y \in Y_{R, t}} \inf _{\phi \in \Phi_{R, N}} \mathcal{E}_{V_{\beta, t}^{M}}\left(S_{t, y}\left(\phi * \delta_{\varepsilon}\right)\right)-2 \varphi(t)^{-2} \eta .
$$

Proof. - Let $f \in H_{0}^{1,2}\left(B_{1}(0)\right),\|f\|_{2}=1$, be any fixed test function. Set $c_{10} \stackrel{\text { def }}{=}\|\nabla f\|_{2}^{2}$ ( $c_{9}$ is defined below). We choose $\beta, \eta$, and $M$. By Lemma 2.1, by the lower bound (2.13), and by scaling we know that for $R=\left(c_{7} / \eta\right)^{1 / 2} \vee 1$ and $t>0$ we have

$$
\lambda_{V_{\beta, t}}\left(\mathcal{T}_{t}\right) \geqslant \min _{y \in Y_{R, t}} \inf _{\substack{\phi \in H_{0}^{1,2}\left(B_{R}(0)\right) \\\|\phi\|_{2}=1}} \mathcal{E}_{V_{\beta, t}^{M}}\left(S_{t, y} \phi\right)-\varphi(t)^{-2} \eta .
$$

For every normalized test function $\phi \in H_{0}^{1,2}\left(B_{R}(0)\right) \backslash \Phi_{R, N}$ we have

$$
\mathcal{E}_{V_{\beta, t}^{M}}\left(S_{t, y} \phi\right)>\frac{1}{2} \varphi(t)^{-2} N \geqslant \frac{1}{2}\left\|\nabla\left(S_{t, y} f\right)\right\|_{2}^{2}+\beta \varphi(t)^{-2} M \geqslant \mathcal{E}_{V_{\beta, t}^{M}}\left(S_{t, y} f\right) .
$$


Hence we can restrict the infimum in (2.15) to the smaller class $\Phi_{R, N}$ :

$$
\lambda_{V_{\beta, t}^{M}}\left(\mathcal{T}_{t}\right) \geqslant \min _{y \in Y_{R, t}} \inf _{\phi \in \Phi_{R, N}} \mathcal{E}_{V_{\beta, t}^{M}}\left(S_{t, y} \phi\right)-\varphi(t)^{-2} \eta
$$

To deal with convolutions, we use the Fourier transform $\hat{\psi}(k) \stackrel{\text { def }}{=} \int_{\mathbb{R}^{d}} \mathrm{e}^{-i k x} \psi(x) \mathrm{d} x$ : There is a constant $c_{9}(d)>0$ such that for all $\varepsilon>0$ and $k \in \mathbb{R}^{d}$ the estimate $\left|1-\hat{\delta}_{\varepsilon}(k)\right| \leqslant c_{9} \varepsilon k$ holds; to see this one observes that $\hat{\delta}_{\varepsilon}(k)=\hat{\delta}_{1}(\varepsilon k), \hat{\delta}_{1}(0)=1$, and by Lipschitz continuity $\left|\hat{\delta}_{1}(0)-\hat{\delta}_{1}(k)\right| \leqslant c_{9}|k|$ for some constant $c_{9}\left(\hat{\delta}_{1}\right.$ is even real analytic, since $\delta_{1}$ is compactly supported, and $\left.\hat{\delta}_{1}(k) \stackrel{|k| \rightarrow \infty}{\longrightarrow} 0\right)$. We estimate:

$$
\left\|\phi-\phi * \delta_{\varepsilon}\right\|_{2}=(2 \pi)^{-d / 2}\left\|\left(1-\hat{\delta}_{\varepsilon}\right) \hat{\phi}\right\|_{2} \leqslant(2 \pi)^{-d / 2} c_{9} \varepsilon\|k \hat{\phi}(k)\|_{2}=c_{9} \varepsilon\|\nabla \phi\|_{2} .
$$

We remark $\left\|\phi * \delta_{\varepsilon}\right\|_{2} \leqslant\|\phi\|_{2}$; this is a consequence of $\left\|\delta_{\varepsilon}\right\|_{1}=1$ and the integral version of the triangle inequality. Therefore we have for all $\phi \in \Phi_{R, N}$ and $\varepsilon \in$ $\left(0, \eta\left(2 \beta M c_{9} N^{1 / 2}\right)^{-1}\right]:$

$$
\begin{aligned}
\left|\int_{\mathbb{R}^{d}} V_{\beta, t}^{M}\left[\left(S_{t, y} \phi\right)^{2}-\left(S_{t, y}\left(\phi * \delta_{\varepsilon}\right)\right)^{2}\right] \mathrm{d} x\right| & \leqslant\left\|V_{\beta, t}^{M}\right\|_{\infty}\left\|\left(S_{t, y} \phi\right)^{2}-\left(S_{t, y}\left(\phi * \delta_{\varepsilon}\right)\right)^{2}\right\|_{1} \\
& =\left\|V_{\beta, t}^{M}\right\|_{\infty}\left\|\left(\phi+\phi * \delta_{\varepsilon}\right)\left(\phi-\phi * \delta_{\varepsilon}\right)\right\|_{1} \\
& \leqslant\left\|V_{\beta, t}^{M}\right\|_{\infty} 2\|\phi\|_{2}\left\|\phi-\phi * \delta_{\varepsilon}\right\|_{2} \\
& \leqslant 2\left\|V_{\beta, t}^{M}\right\|_{\infty} c_{9} \varepsilon\|\nabla \phi\|_{2} \\
& \leqslant 2 \beta M \varphi(t)^{-2} c_{9} \varepsilon N^{1 / 2} \leqslant \varphi(t)^{-2} \eta .
\end{aligned}
$$

Using the integral version of the triangle inequality once more, we see

$$
\left\|\nabla\left(S_{t, y}\left(\phi * \delta_{\varepsilon}\right)\right)\right\|_{2}=\left\|\left(\nabla\left(S_{t, y} \phi\right)\right) * \delta_{\varepsilon \varphi(t)}\right\|_{2} \leqslant\left\|\nabla\left(S_{t, y} \phi\right)\right\|_{2} .
$$

Combining the estimates (2.19) and (2.20) we get

$$
\mathcal{E}_{V_{\beta, t}^{M}}\left(S_{t, y}\left(\phi * \delta_{\varepsilon}\right)\right)-\mathcal{E}_{V_{\beta, t}^{M}}\left(S_{t, y} \phi\right) \leqslant \varphi(t)^{-2} \eta .
$$

Finally we combine this with (2.17) to get the claim (2.14). Lemma 2.2 is proved.

LemmA 2.3. - Given $\beta>0, \eta>0$ and $M>0$, there is $R \geqslant 1$ and a finite set $\Psi(\eta, \beta, M) \subseteq C_{c}^{1}\left(\overline{B_{R+1}(0)}\right)$ of normalized functions (i.e. $\|\psi\|_{2}=1$ for $\left.\psi \in \Psi\right)$ such that for all $t>0$ :

$$
\lambda_{V_{\beta, t}}\left(\mathcal{T}_{t}\right) \geqslant \min _{\substack{y \in Y_{R, t} \\ \psi \in \Psi}} \mathcal{E}_{V_{\beta, t}^{M}}\left(S_{t, y} \psi\right)-3 \varphi(t)^{-2} \eta
$$

Proof. - Choose $R \geqslant 1$ and $N$ by Lemma 2.2. We choose $\varepsilon>0$ so small that the following three conditions hold true:

$$
\varepsilon \leqslant 1, \quad c_{9} \varepsilon N^{1 / 2} \leqslant \frac{1}{2}, \quad 2 c_{9} \varepsilon N^{1 / 2}(2 N+\beta M) \leqslant \frac{\eta}{2} ;
$$


especially Lemma 2.2 is applicable for this choice of $\varepsilon$. Set $R^{\prime} \stackrel{\text { def }}{=} R+1 \geqslant R+\varepsilon$, so the convolution map $\psi \mapsto \psi * \delta_{\varepsilon}$ maps $H_{0}^{1,2}\left(B_{R}(0)\right)$ to $C_{c}^{1}\left(B_{R^{\prime}}(0)\right)$. We endow $C_{c}^{1}\left(B_{R^{\prime}}(0)\right)$ with the norm $\psi \mapsto\|\psi\|_{\infty}+\|\nabla \psi\|_{\infty}$. As a consequence of the Arzelà-Ascoli theorem this convolution map $H_{0}^{1,2}\left(B_{R}(0)\right) \stackrel{* \delta_{\varepsilon}}{\longrightarrow} C_{c}^{1}\left(B_{R^{\prime}}(0)\right)$ is a compact linear operator. Since $\Phi_{R, N}$ is bounded in $H_{0}^{1,2}\left(B_{R}(0)\right)$, its image $\Phi_{R, N} * \delta_{\varepsilon}=\left\{\phi * \delta_{\varepsilon}: \phi \in \Phi_{R, N}\right\}$ is relatively compact in $C_{c}^{1}\left(B_{R^{\prime}}(0)\right)$.

We claim that for every bounded set $S \subseteq C_{c}^{1}\left(B_{R^{\prime}}(0)\right)$ the family of maps

$$
\left(F_{t, y}: S \rightarrow \mathbb{R}\right) \underset{\substack{t>0 \\ y \in Y_{R, t}}}{ }, \quad F_{t, y}(\psi) \stackrel{\text { def }}{=} \varphi(t)^{2} \mathcal{E}_{V_{\beta, t}^{M}}\left(S_{t, y} \psi\right),
$$

is equicontinuous, i.e. for every $\eta>0$ there is an $\alpha>0$ such that for all $t>0, y \in Y_{R, t}$, and $\psi_{1}, \psi_{2} \in S$ :

$$
\left\|\psi_{1}-\psi_{2}\right\|_{\infty}+\left\|\nabla\left(\psi_{1}-\psi_{2}\right)\right\|_{\infty}<\alpha \text { implies }\left|F_{t, y}\left(\psi_{1}\right)-F_{t, y}\left(\psi_{2}\right)\right|<\frac{\eta}{2} .
$$

To prove this claim, we observe first that the inclusion map $C_{c}^{1}\left(\overline{B_{R^{\prime}}(0)}\right) \rightarrow H_{0}^{1,2}\left(B_{R^{\prime}}(0)\right)$ is continuous:

$$
\|\psi\|_{2}+\|\nabla \psi\|_{2} \leqslant c_{11}\|\psi\|_{\infty}+c_{11}\|\nabla \psi\|_{\infty}
$$

for $\psi \in C_{c}^{1}\left(\overline{B_{R^{\prime}}(0)}\right), c_{11} \stackrel{\text { def }}{=}\left|B_{R^{\prime}}(0)\right|^{1 / 2}$. Let $s_{2} \stackrel{\text { def }}{=} \sup \left\{\|\psi\|_{2}+\|\nabla \psi\|_{2}: \psi \in S\right\}<\infty$. We choose $\alpha$ so small that $s_{2}(1+2 \beta M) c_{11} \alpha \leqslant \eta / 2$. Using the Cauchy-Schwarz inequality:

$$
\begin{aligned}
\left|F_{t, y}\left(\psi_{1}\right)-F_{t, y}\left(\psi_{2}\right)\right| & \leqslant \frac{1}{2}\left|\left\|\nabla \psi_{1}\right\|_{2}^{2}-\left\|\nabla \psi_{2}\right\|_{2}^{2}\right|+\beta M\left\|\left(S_{t, y} \psi_{1}\right)^{2}-\left(S_{t, y} \psi_{2}\right)^{2}\right\|_{1} \\
& \leqslant \frac{1}{2}\left\|\nabla\left(\psi_{1}+\psi_{2}\right)\right\|_{2}\left\|\nabla\left(\psi_{1}-\psi_{2}\right)\right\|_{2}+\beta M\left\|\psi_{1}^{2}-\psi_{2}^{2}\right\|_{1} \\
& \leqslant 2 s_{2}\left(\frac{1}{2}\left\|\nabla\left(\psi_{1}-\psi_{2}\right)\right\|_{2}+\beta M\left\|\psi_{1}-\psi_{2}\right\|_{2}\right) \\
& \leqslant s_{2}(1+2 \beta M) c_{11} \alpha \leqslant \frac{\eta}{2}
\end{aligned}
$$

Combining the relative compactness of $\Phi_{R, N} * \delta_{\varepsilon}$ with the equicontinuity (2.25) we obtain the following: there is a finite set $\tilde{\Psi} \subseteq \Phi_{R, N} * \delta_{\varepsilon}$ such that for all $t>0$ and $y \in Y_{R, t}$ we have the lower bound

$$
\inf _{\phi \in \Phi_{R, N}} F_{t, y}\left(\phi * \delta_{\varepsilon}\right) \geqslant \min _{\psi \in \tilde{\Psi}} F_{t, y}(\psi)-\frac{\eta}{2} .
$$

We normalize these test functions: $\Psi \stackrel{\text { def }}{=}\left\{\|\psi\|_{2}^{-1} \psi: \psi \in \tilde{\Psi}\right\}$; this is well defined, since for $\psi=\phi * \delta_{\varepsilon}, \phi \in \Phi_{R, N}$ we have

$$
\left|\|\psi\|_{2}-1\right| \leqslant\|\psi-\phi\|_{2} \leqslant c_{9} \varepsilon N^{1 / 2} \leqslant \frac{1}{2}
$$

by (2.18) in the second step and (2.23) in the last step. A quantitative bound on $F_{t, y}\left(\|\psi\|_{2}^{-1} \psi\right)$ for these functions $\psi \in \Phi_{R, N} * \delta_{\varepsilon}$ is (using the integral version of the 
triangle inequality):

$$
F_{t, y}\left(\|\psi\|_{2}^{-1} \psi\right) \leqslant \frac{1}{2} \frac{\|\nabla \psi\|_{2}^{2}}{\|\psi\|_{2}^{2}}+\beta M \leqslant 2 N+\beta M .
$$

We estimate, using the bounds (2.28), (2.29), (2.30), and (2.23):

$$
\begin{aligned}
\inf _{\phi \in \Phi_{R, N}} F_{t, y}\left(\phi * \delta_{\varepsilon}\right) & \geqslant \min _{\psi \in \tilde{\Psi}}\|\psi\|_{2}^{2} F_{t, y}\left(\|\psi\|_{2}^{-1} \psi\right)-\frac{\eta}{2} \geqslant\left(1-c_{9} \varepsilon N^{1 / 2}\right)^{2} \min _{\psi \in \Psi} F_{t, y}(\psi)-\frac{\eta}{2} \\
& \geqslant \min _{\psi \in \Psi} F_{t, y}(\psi)-2 c_{9} \varepsilon N^{1 / 2}(2 N+\beta M)-\frac{\eta}{2} \\
& \geqslant \min _{\psi \in \Psi} F_{t, y}(\psi)-\eta .
\end{aligned}
$$

Lemma 2.3 follows now from the bounds (2.14), (2.31) and definition (2.24) of $F_{t, y}$.

We discretize the space $\mathbb{R}^{d}$ on a very fine scale $\zeta \in\left(0, d^{-1 / 2} a\right)$ (to be specified later; it is smaller than the diameter of the shape functions): Set $K_{j}(\zeta) \stackrel{\text { def }}{=} j+[0, \zeta)^{d}, j \in \zeta \mathbb{Z}^{d}$. We define the i.i.d. Bernoulli variables $\xi_{j}(\zeta) \stackrel{\text { def }}{=} 1_{\left\{\omega\left(K_{j}\right) \geqslant 1\right\}}$ and a discretized version $\omega^{\zeta}$ of the Poissonian cloud configuration $\omega$ by

$$
\omega^{\zeta} \stackrel{\text { def }}{=} \sum_{j \in \zeta \mathbb{Z}^{d}} \xi_{j} \delta_{j}
$$

in this equation $\delta_{j}$ is the Dirac measure located at $j$. We observe

$$
\mathbb{P}\left[\xi_{j}=0\right]=\mathbb{P}\left[\omega^{\zeta}\left(K_{j}\right)=0\right]=1-\mathbb{P}\left[\omega^{\zeta}\left(K_{j}\right)=1\right]=\mathrm{e}^{-\zeta^{d}} .
$$

Finally we set

$$
M(\zeta) \stackrel{\text { def }}{=} W_{\infty}\left|B_{2 a}(0)\right| \zeta^{-d} .
$$

We remark that $M=M(\zeta)$ is finite since the shape function $W$ is bounded; finiteness of $M$ played a role in the proof of Lemma 2.3, above.

We define an unscaled and a scaled lattice version of the potential:

$$
\tilde{V}^{\zeta}(x) \stackrel{\text { def }}{=} \int_{\mathbb{R}^{d}} W(x-y) \omega^{\zeta}(\mathrm{d} y) \quad \text { and } \quad \tilde{V}_{\beta, t}^{\zeta, \varphi}(x) \stackrel{\text { def }}{=} \beta \varphi(t)^{-2} \tilde{V}^{\zeta}(x) .
$$

The next lemma compares the two potentials $V_{\beta, t}^{M}$ and $\tilde{V}_{\beta, t}^{\zeta}$ :

Lemma 2.4. - Given $\beta>0, \eta>0$ and $\zeta \in\left(0, d^{-1 / 2} a\right)$, define $M$ by (2.34), and let $R \geqslant 1$ and $\Psi$ be chosen according to Lemma 2.3. Then

$$
\liminf _{t \rightarrow \infty} \min _{\substack{y \in Y_{R, t} \\ \psi \in \Psi}} \varphi(t)^{2}\left(\mathcal{E}_{V_{\beta, t}^{M}}\left(S_{t, y} \psi\right)-\mathcal{E}_{\tilde{V}_{\beta, t}^{\zeta}}\left(S_{t, y} \psi\right)\right) \geqslant 0 .
$$

Proof. - Let $s_{\infty} \stackrel{\text { def }}{=} \max _{\psi \in \Psi}\left(\|\psi\|_{\infty}+\|\nabla \psi\|_{\infty}\right)<\infty$, then the functions $\left(S_{t, y} \psi\right)^{2}$ with $t>0, y \in Y_{R, t}, \psi \in \Psi$, are Lipschitz continuous: 


$$
\begin{aligned}
\left|\left(S_{t, y} \psi\right)\left(x_{1}\right)^{2}-\left(S_{t, y} \psi\right)\left(x_{2}\right)^{2}\right| & \leqslant 2\left\|S_{t, y} \psi\right\|_{\infty}\left\|\nabla\left(S_{t, y} \psi\right)\right\|_{\infty}\left|x_{1}-x_{2}\right| \\
& \leqslant 2 s_{\infty}^{2} \varphi(t)^{-d-1}\left|x_{1}-x_{2}\right| .
\end{aligned}
$$

We use the notation $W^{-}(x) \stackrel{\text { def }}{=} W(-x)$. Using $\|W\|_{1}=1$ we see that the functions $\left(S_{t, y} \psi\right)^{2} * W^{-}$are also Lipschitz continuous with the same upper bound $2 s_{\infty}^{2} \varphi(t)^{-d-1}$ for the Lipschitz constant. Let $\hat{\omega}^{\zeta}$ denote any point configuration with $\hat{\omega}^{\zeta} \leqslant \omega$ and $\hat{\omega}^{\zeta}\left(K_{j}\right)=\omega^{\zeta}\left(K_{j}\right)$; this means that $\hat{\omega}^{\zeta}$ is obtained from $\omega$ by removing extra points of the Poissonian cloud $\omega$ in boxes $K_{j}$ with $\omega\left(K_{j}\right)>1$. The choice (2.34) of $M$ guarantees

$$
V^{M}(x) \geqslant \int_{\mathbb{R}^{d}} W(x-y) \hat{\omega}^{\zeta}(\mathrm{d} y) .
$$

Using $\hat{\omega}^{\zeta}\left(K_{j}\right)=\omega^{\zeta}\left(K_{j}\right) \leqslant 1$ we conclude

$$
\int_{K_{j}}\left(S_{t, y} \psi\right)^{2} * W^{-} \mathrm{d} \omega^{\zeta}-\int_{K_{j}}\left(S_{t, y} \psi\right)^{2} * W^{-} \mathrm{d} \hat{\omega}^{\zeta} \leqslant 2 s_{\infty}^{2} \varphi(t)^{-d-1} \sqrt{d} \zeta .
$$

$\left(S_{t, y} \psi\right)^{2} * W^{-}$is supported in a ball of radius $(R+1) \varphi(t)+a \leqslant 3 R \varphi(t)$ (for large $t$ ), and the union of all $K_{j}$ that have a non-empty intersection with this ball is contained in a ball of radius $4 R \varphi(t)$ (for large t). Therefore the number of these $K_{j}$ 's is at most $\left|B_{4 R \varphi(t)}(0)\right| / \zeta^{d}$. We estimate for large $t$, using (2.38), (2.39) and Fubini's theorem,

$$
\begin{aligned}
& \varphi(t)^{2}\left(\mathcal{E}_{\tilde{V}_{\beta, t}^{\zeta}}\left(S_{t, y} \psi\right)-\mathcal{E}_{V_{\beta, t}^{M}}\left(S_{t, y} \psi\right)\right) \\
& \quad \leqslant \beta \int_{\mathbb{R}^{d}}\left(S_{t, y} \psi\right)^{2} * W^{-} \mathrm{d} \omega^{\zeta}-\beta \int_{\mathbb{R}^{d}}\left(S_{t, y} \psi\right)^{2} * W^{-} \mathrm{d} \hat{\omega}^{\zeta} \\
& \quad \leqslant \beta \cdot 2 s_{\infty}^{2} \sqrt{d} \zeta^{1-d} R^{d}\left|B_{4}(0)\right| \varphi(t)^{-1} \stackrel{t \rightarrow \infty}{\longrightarrow} 0,
\end{aligned}
$$

since $\varphi(t) \rightarrow \infty$ as $t \rightarrow \infty$. Lemma 2.4 is proved.

We define a discretized version of the Lebesgue measure

$$
v^{\zeta} \stackrel{\text { def }}{=} \zeta^{d} \sum_{j \in \zeta \mathbb{Z}^{d}} \delta_{j}
$$

compare this with definition (2.32). The map $\zeta \mapsto \zeta^{-d}\left(1-\mathrm{e}^{-\zeta^{d}}\right)$ maps the interval $(0, \infty)$ diffeomorphically and monotonically decreasing onto the interval $(0,1)$; especially we have $\zeta^{-d}\left(1-\mathrm{e}^{-\zeta^{d}}\right) \stackrel{\zeta \rightarrow 0}{\longrightarrow} 1$. We define $\zeta(m, d)$ for $0<m<1$ implicitly by the equation

$$
m=\zeta^{-d}\left(1-\mathrm{e}^{-\zeta^{d}}\right)
$$

We define $\sigma_{t} \stackrel{\text { def }}{=} \varphi(t)^{d} \sigma, \zeta_{t} \stackrel{\text { def }}{=} \varphi(t)^{-1} \zeta, y_{t} \stackrel{\text { def }}{=} \varphi(t)^{-1} y$, and $W_{t}(x) \stackrel{\text { def }}{=} \varphi(t)^{d} W(\varphi(t) x) . W_{t}$ is supported in $B_{a \varphi(t)^{-1}}(0)$ and fulfills $\left\|W_{t}\right\|_{1}=\|W\|_{1}=1$. Let $T_{z} v$ denote the translation 
of a measure $v$ by $z \in \mathbb{R}^{d}$, i.e. $\left(T_{z} v\right)(A)=v(A-z)$. Further for $\psi \in \Phi$ we define

$$
o_{\psi, \sigma, t} \stackrel{\text { def }}{=} \sup _{z \in \mathbb{R}^{d}}\left|\int_{\mathbb{R}^{d}}\left(\exp \left\{\sigma \psi^{2} * W_{t}^{-}\right\}-1\right) \mathrm{d}\left(T_{z} v^{\zeta_{t}}\right)-\Lambda_{\psi}(\sigma)\right| .
$$

LEMMA 2.5. - Let $\beta>0, \eta>0$, and assume that $m<1$ is so close to 1 that $\zeta \in\left(0, d^{-1 / 2} a\right)$. Choose $R$ and $\Psi$ as in Lemma 2.4. Then for all $\psi \in \Psi, \mu \geqslant 0, \sigma \leqslant 0$ and $t>0$

$$
\begin{aligned}
& \mathbb{P}\left[\min _{y \in Y_{R, t}} \int_{\mathbb{R}^{d}} \tilde{V}^{\zeta} \cdot\left(S_{t, y} \psi\right)^{2} \mathrm{~d} x \leqslant m \mu\right] \\
& \quad \leqslant \exp \left\{\log \left|Y_{R, t}\right|-m \varphi(t)^{d}\left(\sigma \mu-\Lambda_{\psi}(\sigma)-o_{\psi, \sigma, t}\right)\right\} .
\end{aligned}
$$

Proof. - We need some preparations for the large deviation estimates in the derivation of (2.44): Let $f$ be any compactly supported bounded measurable function. Using independence, (2.33), and $\log (1+x) \leqslant x$ we have

$$
\begin{aligned}
\mathbb{E}\left[\exp \left\{\sigma \int_{\mathbb{R}^{d}} f \mathrm{~d} \omega^{\zeta}\right\}\right] & =\prod_{j \in \zeta \mathbb{Z}^{d}}\left(1+\left(1-\mathrm{e}^{-\zeta^{d}}\right)\left(\mathrm{e}^{\sigma f(j)}-1\right)\right) \\
& \leqslant \exp \left\{m \int_{\mathbb{R}^{d}}\left(\mathrm{e}^{\sigma f}-1\right) \mathrm{d} v^{\zeta}\right\} .
\end{aligned}
$$

Choose $\psi \in \Psi, \mu \geqslant 0, \sigma \leqslant 0$ and $t>0$. Using the exponential Chebyshev inequality and a change of variables $x \mapsto \varphi(t)^{-1}(x-y)$ in the following large deviation estimate ( $\sigma_{t}$ is non-positive) we obtain

$$
\begin{aligned}
& \mathbb{P}\left[\min _{y \in Y_{R, t}} \int_{\mathbb{R}^{d}} \tilde{V}^{\zeta} \cdot\left(S_{t, y} \psi\right)^{2} \mathrm{~d} x \leqslant m \mu\right]=\mathbb{P}\left[\min _{y \in Y_{R, t}} \int_{\mathbb{R}^{d}}\left(S_{t, y} \psi\right)^{2} * W^{-} \mathrm{d} \omega^{\zeta} \leqslant m \mu\right] \\
& \leqslant \sum_{y \in Y_{R, t}} \mathbb{P}\left[\int_{\mathbb{R}^{d}}\left(S_{t, y} \psi\right)^{2} * W^{-} \mathrm{d} \omega^{\zeta} \leqslant m \mu\right] \\
& \leqslant \sum_{y \in Y_{R, t}} \mathrm{e}^{-\sigma_{t} m \mu} \mathbb{E}\left[\exp \left\{\sigma_{t} \int_{\mathbb{R}^{d}}\left(S_{t, y} \psi\right)^{2} * W^{-} \mathrm{d} \omega^{\zeta}\right\}\right] \\
& \leqslant \sum_{y \in Y_{R, t}} \exp \left\{-\sigma_{t} m \mu+m \int_{\mathbb{R}^{d}}\left(\exp \left\{\sigma_{t}\left(S_{t, y} \psi\right)^{2} * W^{-}\right\}-1\right) \mathrm{d} \nu^{\zeta}\right\} \\
& =\sum_{y \in Y_{R, t}} \exp \left\{-m \varphi(t)^{d}\left(\sigma \mu-\int_{\mathbb{R}^{d}}\left(\exp \left\{\sigma \psi^{2} * W_{t}^{-}\right\}-1\right) \mathrm{d}\left(T_{y_{t}} \nu^{\zeta t}\right)\right)\right\} \\
& \leqslant \exp \left\{\log \left|Y_{R, t}\right|-m \varphi(t)^{d}\left(\sigma \mu-\Lambda_{\psi}(\sigma)-o_{\psi, \sigma, t}\right)\right\} .
\end{aligned}
$$

This finishes the proof of Lemma 2.5. 


\subsection{Proof of the lower bound in Theorem 0.1}

In this subsection we always assume that $\varphi(t)=(\log t)^{1 / d}$ for $t>1$.

LEMMA 2.6. - Let $d \geqslant 1, \varphi(t)=(\log t)^{1 / d}, \beta>0, \eta>0$, and assume that $m<1$ is so close to 1 that $\zeta \in\left(0, d^{-1 / 2} a\right)$. Choose $R$ and $\Psi$ as in Lemma 2.4. Then for all $\psi \in \Psi$ and $\mu \in[0,1)$ with

$$
\Lambda_{\psi}^{*}(\mu)>\frac{d}{m}
$$

there exist $\vartheta>0$ and $t_{0}>1$ such that for all $t>t_{0}$ :

$$
\mathbb{P}\left[\min _{y \in Y_{R, t}} \int_{\mathbb{R}^{d}} \tilde{V}^{\zeta} \cdot\left(S_{t, y} \psi\right)^{2} \mathrm{~d} x \leqslant m \mu\right] \leqslant t^{-\vartheta} .
$$

Proof. - Choose $\psi \in \Psi$ and $\mu$ such that (2.47) is fulfilled. By definition (0.5) of $\Lambda_{\psi}^{*}$, there is $\sigma \in \mathbb{R}$ with

$$
\sigma \mu-\Lambda_{\psi}(\sigma)>\frac{d}{m}
$$

We may even choose $\sigma \leqslant 0$ (to see this, one proceeds as follows: for $\sigma \geqslant 0, \mu \in[0,1$ ),

$$
\frac{\mathrm{d}}{\mathrm{d} \sigma}\left(\mu \sigma-\Lambda_{\psi}(\sigma)\right)=\mu-\int_{\mathbb{R}^{d}} \psi^{2} \mathrm{e}^{\sigma \psi^{2}} \mathrm{~d} x \leqslant \mu-\|\psi\|_{2}^{2}=\mu-1<0,
$$

especially we get for $\sigma \geqslant \sigma_{0}=0$ that $\left.\sigma_{0} \mu-\Lambda_{\psi}\left(\sigma_{0}\right) \geqslant \sigma \mu-\Lambda_{\psi}(\sigma)\right)$. We set

$$
\vartheta \stackrel{\text { def }}{=} \frac{1}{2}\left[m\left(\sigma \mu-\Lambda_{\psi}(\sigma)\right)-d\right]>0,
$$

where $\sigma \leqslant 0$ fulfills (2.49). By the Lipschitz continuity of $\psi$ and the dominated convergence theorem we know that

$$
o_{\psi, \sigma, t} \stackrel{t \rightarrow \infty}{\longrightarrow} 0
$$

so Lemma 2.6 is a consequence of Lemma 2.5, of the asymptotics

$$
\frac{\log \left|Y_{R, t}\right|}{\log t} \stackrel{t \rightarrow \infty}{\longrightarrow} d
$$

and of the choice (2.51) of $\vartheta$.

LEMMA 2.7. - For $d \geqslant 1, \varphi(t)=(\log t)^{1 / d}$, and $\beta>0$ the following holds $\mathbb{P}$-a.s.:

$$
\liminf _{t \rightarrow \infty}(\log t)^{2 / d} \lambda_{V_{\beta, t}}\left(\mathcal{T}_{t}\right) \geqslant I_{1}(\beta) .
$$

Proof. - Let $\beta>0, \eta>0$. Take $m<1$ so close to 1 that the following three assumptions are fulfilled: (i) Lemma 2.6 is applicable, (ii) (recall $I_{1}=I_{2} \leqslant \beta$, see 
Corollary 1.2 and (1.8))

$$
I_{1}-\eta<\beta m,
$$

and (iii) (recall (1.3) and (1.8))

$$
I_{1}<\tilde{I}_{1}(\beta, m)+\eta \leqslant \frac{1}{2}\|\nabla \phi\|_{2}^{2}+\beta m \mu+\eta
$$

holds for all $\phi \in \Phi$ and $\mu \in(0,1)$ with

$$
\Lambda_{\phi}^{*}(\mu) \leqslant \frac{d}{m} .
$$

Choose $\zeta, M$ and $R, \Psi \subseteq \Phi$ as in Lemmas 2.6, 2.3, 2.4, respectively. Then we get for large $t$, using (2.22), (2.36), definition (0.2), and the scaling property (2.11):

$$
\begin{aligned}
(\log t)^{2 / d} \lambda_{V_{\beta, t}}\left(\mathcal{T}_{t}\right) & \geqslant-3 \eta+(\log t)^{2 / d} \min _{\substack{y \in Y_{R, t} \\
\psi \in \Psi}} \mathcal{E}_{V_{\beta, t}^{M}}\left(S_{t, y} \psi\right) \\
& \geqslant-4 \eta+(\log t)^{2 / d} \min _{\substack{y \in Y_{R, t} \\
\psi \in \Psi}} \mathcal{E}_{\tilde{V}_{\beta, t}^{\zeta}}\left(S_{t, y} \psi\right) \\
& =-4 \eta+\min _{\psi \in \Psi}\left(\frac{1}{2}\|\nabla \psi\|^{2}+\beta \min _{y \in Y_{R, t}} \int_{\mathbb{R}^{d}} \tilde{V}^{\zeta} \cdot\left(S_{t, y} \psi\right)^{2} \mathrm{~d} x\right) .
\end{aligned}
$$

For all $\psi \in \Psi$ we define

$$
\mu_{\psi} \stackrel{\text { def }}{=}\left(I_{1}-\frac{1}{2}\|\nabla \psi\|^{2}-\eta\right)(\beta m)^{-1}<1,
$$

see (2.55). We define the finite set $\Xi \stackrel{\text { def }}{=}\left\{\psi \in \Psi: \mu_{\psi}>0\right\}$. We compare

$$
I_{1}=\frac{1}{2}\|\nabla \psi\|^{2}+\beta m \mu_{\psi}+\eta
$$

with (2.56): There are two cases to distinguish:

Case 1. If $\mu_{\psi} \notin \Xi$, then we get trivially for all $t>1$ :

$$
\min _{y \in Y_{R, t}} \int_{\mathbb{R}^{d}} \tilde{V}^{\zeta} \cdot\left(S_{t, y} \psi\right)^{2} \mathrm{~d} x \geqslant 0 \geqslant m \mu_{\psi} .
$$

Case 2. Else if $\mu_{\psi} \in \Xi$, i.e. $0<\mu_{\psi}<1$; then the condition (2.57) must fail (compare (2.56) and (2.60)), i.e. $\Lambda_{\psi}^{*}\left(\mu_{\psi}\right)>\frac{d}{m}$. In this case we apply Lemma 2.6: it provides a $\vartheta_{\psi}>0$ such that for all large $t$

$$
\mathbb{P}\left[\min _{y \in Y_{R, t}} \int_{\mathbb{R}^{d}} \tilde{V}^{\zeta} \cdot\left(S_{t, y} \psi\right)^{2} \mathrm{~d} x \geqslant m \mu_{\psi}\right] \geqslant 1-t^{-\vartheta_{\psi}} .
$$


Collecting both cases we get for all large $t$, using (2.58) and (2.60):

$$
\begin{aligned}
& \mathbb{P}\left[(\log t)^{2 / d} \lambda_{V_{\beta, t}}\left(\mathcal{T}_{t}\right) \geqslant I_{1}-5 \eta\right] \\
& \quad \geqslant \mathbb{P}\left[\min _{\psi \in \Psi}\left(\frac{1}{2}\|\nabla \psi\|^{2}+\beta \min _{y \in Y_{R, t}} \int_{\mathbb{R}^{d}} \tilde{V}^{\zeta} \cdot\left(S_{t, y} \psi\right)^{2} \mathrm{~d} x\right) \geqslant I_{1}-\eta\right] \\
& \quad \geqslant \mathbb{P}\left[\min _{\substack{y \in Y_{R, t} \\
\psi \in \Psi}}\left(\int_{\mathbb{R}^{d}} \tilde{V}^{\zeta} \cdot\left(S_{t, y} \psi\right)^{2} \mathrm{~d} x-m \mu_{\psi}\right) \geqslant 0\right] \geqslant 1-\sum_{\psi \in \Xi} t^{-\vartheta_{\psi}} .
\end{aligned}
$$

We choose a $\theta \in\left(0, \min _{\psi \in \Xi} \vartheta_{\psi}\right)$ and define the increasing sequence $t_{n}=n^{2 / \theta} \stackrel{n \rightarrow \infty}{\longrightarrow} \infty$; then $\sum_{n \in \mathbb{N}} \sum_{\psi \in \Xi} t_{n}^{-\vartheta_{\psi}}<\infty$. The Borel-Cantelli lemma and (2.64) imply that

$$
\mathbb{P} \text {-a.s. } \quad \liminf _{n \rightarrow \infty}\left(\log t_{n}\right)^{2 / d} \lambda_{V_{\beta, t_{n}}}\left(\mathcal{T}_{t_{n}}\right) \geqslant I_{1}-5 \eta .
$$

For $t>1$, let $n(t)$ denote the smallest index with $t_{n(t)} \geqslant t$. Since $\lambda_{V_{\beta, t}}\left(\mathcal{T}_{t}\right) \geqslant \lambda_{V_{\beta, t_{n}(t)}}\left(\mathcal{T}_{t_{n(t)}}\right)$ and $(\log t) /\left(\log t_{n(t)}\right) \stackrel{t \rightarrow \infty}{\longrightarrow} 1$, we see that

$$
\mathbb{P} \text {-a.s. } \quad \liminf _{t \rightarrow \infty}(\log t)^{2 / d} \lambda_{V_{\beta, t}}\left(\mathcal{T}_{t}\right) \geqslant I_{1}-5 \eta .
$$

But now the claim of Lemma 2.7 follows because $\eta>0$ was chosen arbitrarily.

Proof of Theorem 0.1. - Theorem 0.1 is now proven, too: It follows from formula (1.7), Corollary 1.2 and Lemma 2.7.

\subsection{Proof of the lower bound in Theorem 0.6}

The following lemma is analogous to Lemma 2.6 but with a different scaling:

LEMMA 2.8. - Let $d \geqslant 1, \varphi(t) \gg(\log t)^{1 / d}, \beta>0, \eta>0$, and assume that $m<1$ is so close to 1 that $\zeta \in\left(0, d^{-1 / 2} a\right)$. Choose $R$ and $\Psi$ as in Lemma 2.4. Then for all $\psi \in \Psi, \mu \in(0,1)$ and $\vartheta>0$ there is a $t_{0}^{\psi}>0$ such that for all $t>t_{0}^{\psi}$ :

$$
\mathbb{P}\left[\min _{y \in Y_{R, t}} \int_{\mathbb{R}^{d}} \tilde{V}^{\zeta} \cdot\left(S_{t, y} \psi\right)^{2} \mathrm{~d} x \leqslant m \mu\right] \leqslant t^{-\vartheta} .
$$

Proof. - Choose $\psi \in \Psi$. The function $\Lambda_{\psi}^{*}$ is convex with the global minimum $\Lambda_{\psi}^{*}(1)=0$ (see Lemma 3.1 of [4]). Hence for any $\mu<1$ there exists $\sigma<0$ such that $\varsigma \stackrel{\text { def }}{=} \sigma \mu-\Lambda_{\psi}(\sigma)>0$ (see also (2.50)). Using Lemma 2.5 we obtain for all $t>0$ :

$$
\mathbb{P}\left[\min _{y \in Y_{R, t}} \int_{\mathbb{R}^{d}} \tilde{V}^{\zeta} \cdot\left(S_{t, y} \psi\right)^{2} \mathrm{~d} x \leqslant m \mu\right] \leqslant \exp \left\{\log \left|Y_{R, t}\right|-m \varphi(t)^{d}\left(\varsigma-o_{\psi, \sigma, t}\right)\right\} .
$$

The bound $\log \left|Y_{R, t}\right| \leqslant d \log t$ is valid for large $t$; consequently $\log \left|Y_{R, t}\right| \ll \varphi(t)^{d}$. Furthermore $o_{\psi, \sigma, t} \stackrel{t \rightarrow \infty}{\longrightarrow} 0$ holds (see also (2.52)). These facts and (2.67) imply the claim. 
Proof of the lower bound in Theorem 0.6. - We choose $\beta>0, \eta \in(0,1)$, and assume that $m \in(1-\eta, 1)$ is so close to 1 that $\zeta \in\left(0, d^{-1 / 2} a\right)$. Choose $R$ and $\Psi$ as in Lemma 2.4. Using Lemmas 2.3 and 2.4 we know that there exists $t_{1}>0$ such that for all $t>t_{1}$

$$
\begin{aligned}
\varphi(t)^{2} \lambda_{V_{\beta, t}}\left(\mathcal{T}_{t}\right) & \geqslant \min _{\substack{y \in Y_{R, t} \\
\psi \in \Psi}} \varphi(t)^{2} \mathcal{E}_{\tilde{V}_{\beta, t}^{\zeta}}\left(S_{t, y} \psi\right)-4 \eta \\
& \geqslant \min _{\substack{y \in Y_{R, t} \\
\psi \in \Psi}} \beta \int_{\mathbb{R}^{d}} \tilde{V}^{\zeta} \cdot\left(S_{t, y} \psi\right)^{2} \mathrm{~d} x-4 \eta .
\end{aligned}
$$

Choose $\vartheta>0$ and $\mu \in(1-\eta, 1)$ (hence $\left.(1-\eta)^{2}<m \mu\right)$. Using Lemma 2.8 we have for all $t \geqslant t_{1} \vee \max _{\psi \in \Psi} t_{0}^{\psi}$

$$
\begin{aligned}
\mathbb{P} & {\left[\varphi(t)^{2} \lambda_{V_{\beta, t}}\left(\mathcal{T}_{t}\right) \geqslant \beta-\eta(2 \beta+4)\right] } \\
& \geqslant \mathbb{P}\left[\min _{\substack{y \in Y_{R, t} \\
\psi \in \Psi}} \int_{\mathbb{R}^{d}} \tilde{V}^{\zeta}\left(S_{t, y} \psi\right)^{2} \mathrm{~d} x \geqslant(1-\eta)^{2}\right] \geqslant 1-\sum_{\psi \in \Psi} t^{-\vartheta} .
\end{aligned}
$$

Hence

$$
\mathbb{P} \text {-a.s. } \quad \liminf _{t \rightarrow \infty} \varphi(t)^{2} \lambda_{V_{\beta, t}^{\varphi}}\left(\mathcal{T}_{t}\right) \geqslant \beta,
$$

this follows by the Borel-Cantelli lemma and since $\eta \in(0,1)$ was chosen arbitrarily (see also (2.64)-(2.65)).

\section{Analysis of the variational principle}

\subsection{The phase transition picture}

We start with citing some well-known facts on the logarithmic moment generating function and the Fenchel-Legendre transform (see Lemma 3.1 and formula (3.6) of [4]): Assume that $\|\phi\|_{2}=1$, then $\Lambda_{\phi}^{*}$ is convex, non-negative, monotonically decreasing on $(0,1)$ with the global minimum $\Lambda_{\phi}^{*}(1)=0$, and for $\mu \in(0,1)$ the maximizing $\sigma$ is nonpositive (see (2.50)) and given by

$$
\mu=\Lambda_{\phi}^{\prime}(\sigma)=\int_{\mathbb{R}^{d}} \phi^{2} \mathrm{e}^{\sigma \phi^{2}} \mathrm{~d} x \quad \text { and } \quad \Lambda_{\phi}^{* \prime}(\mu)=\Lambda_{\phi}^{\prime-1}(\mu)=\sigma .
$$

We set

$$
\Lambda^{*}(\mu) \stackrel{\text { def }}{=} \inf \left\{\Lambda_{\phi}^{*}(\mu): \phi \in \Phi,\|\nabla \phi\|_{2}=1\right\} .
$$

Recall that $\|\phi\|_{2}=1$ holds for all $\phi \in \Phi$.

LEMMA 3.1. - Set $c_{12}(d) \stackrel{\text { def }}{=} d^{-2 / d} / 2$. Then

$$
I(\beta)=\inf _{0<\mu<1}\left(c_{12} \Lambda^{*}(\mu)^{2 / d}+\beta \mu\right) .
$$

Consequently, the function I is concave. 
Proof. - We apply a similar scaling argument as in Lemma 1.1; recall definition (1.14) of the scaled version $\phi_{r}$ of $\phi$, which fulfills $\phi_{r} \in \Phi$ if and only if $\phi \in \Phi$. As a consequence of the scaling properties (1.15), a function $\psi \in H_{0}^{1,2}\left(\mathbb{R}^{d}\right)$ fulfills $\psi \in \Phi$ if and only if there are $r>0$ and $\phi \in \Phi$ with $\|\nabla \phi\|_{2}^{2}=1$ such that $\psi=\phi_{r}$; namely one takes $r=1 /\|\nabla \psi\|_{2}$ and $\phi=\psi_{1 / r}$. This allows us to rewrite $I(\beta)$, using the definition (0.6) of $I$, the facts on $\Lambda_{\phi}^{*}$, and the scaling properties (1.15)-(1.16) again:

$$
\begin{aligned}
I(\beta) & =\inf \left\{\frac{1}{2}\|\nabla \psi\|_{2}^{2}+\beta \mu: \psi \in \Phi, \mu \in(0,1), \Lambda_{\psi}^{*}(\mu) \leqslant d\right\} \\
& =\inf \left\{\frac{1}{2}\left\|\nabla \phi_{r}\right\|_{2}^{2}+\beta \mu: r>0, \phi \in \Phi,\|\nabla \phi\|_{2}^{2}=1, \mu \in(0,1), \Lambda_{\phi_{r}}^{*}(\mu) \leqslant d\right\} \\
& =\inf \left\{\frac{1}{2} r^{-2}+\beta \mu: r>0, \phi \in \Phi,\|\nabla \phi\|_{2}^{2}=1, \mu \in(0,1), r^{d} \Lambda_{\phi}^{*}(\mu) \leqslant d\right\} \\
& =\inf \left\{\frac{1}{2} r^{-2}+\beta \mu: r>0, \phi \in \Phi,\|\nabla \phi\|_{2}^{2}=1, \mu \in(0,1), r^{d} \Lambda_{\phi}^{*}(\mu)=d\right\} \\
& =\inf \left\{\frac{1}{2}\left(\frac{\Lambda_{\phi}^{*}(\mu)}{d}\right)^{2 / d}+\beta \mu: \phi \in \Phi,\|\nabla \phi\|_{2}^{2}=1, \mu \in(0,1)\right\} \\
& =\inf _{0<\mu<1}\left(c_{12} \Lambda^{*}(\mu)^{2 / d}+\beta \mu\right) .
\end{aligned}
$$

The function $I$ is therefore a infimum over linear functions; hence it is concave.

Here is a simple monotonicity argument to get lower bounds for $I(\beta)$ :

Lemma 3.2. - Assume that $\Lambda_{\phi}(\sigma) \leqslant f(\sigma)$ for all $\sigma \leqslant 0, \phi \in \Phi,\|\nabla \phi\|_{2}^{2}=1$. Then for all $\mu \in(0,1)$ :

$$
\Lambda^{*}(\mu) \geqslant f^{*}(\mu)
$$

where $f^{*}(\mu) \stackrel{\text { def }}{=} \sup _{\sigma \leqslant 0}(\mu \sigma-f(\sigma))$. As a consequence we get

$$
I(\beta) \geqslant \inf _{0<\mu<1}\left(c_{12} f^{*}(\mu)^{2 / d}+\beta \mu\right) .
$$

Proof. - Using (2.50) we see that we can restrict the supremum in definition (0.5) of $\Lambda_{\phi}^{*}$ to non-positive values of $\sigma$ whenever $\mu<1$ :

$$
\Lambda_{\phi}^{*}(\mu)=\sup _{\sigma \leqslant 0}\left(\sigma \mu-\Lambda_{\phi}(\sigma)\right) .
$$

This together with the assumptions on $f$ implies (3.5). The lower bound (3.6) then follows from (3.3).

First we provide the lower bound for $d<4$ in (0.10) (compare this with Lemma 3.4 of [4]).

Proof of the lower bound in Theorem 0.3. - Choose $\phi \in \Phi$ with $\|\nabla \phi\|_{2}=1$ and $\|\phi\|_{2}=1$. We use the following Sobolev inequality: For $d<4$ there exists a constant $c_{13}(d)>0$ such that

$$
\|\phi\|_{4}^{4} \leqslant c_{13}(d)
$$


(To see (3.8) for $d=1$, one uses Theorem 8.5(i) in [3], which states $\left\|\phi^{\prime}\right\|_{2}^{2}+\|\phi\|_{2}^{2} \geqslant$ $2\|\phi\|_{\infty}^{2}$, and $\|\phi\|_{4}^{4} \leqslant\|\phi\|_{\infty}^{2}\|\phi\|_{2}^{2}$.

For $d=2$, formula (3.8) is a special case of Theorem 8.5(ii) (3) in [3], which states $\|\nabla \phi\|_{2}^{2}+\|\phi\|_{2}^{2} \geqslant S_{2, q}\|\phi\|_{q}^{2}$ for $2 \leqslant q<\infty, \phi \in H_{0}^{1,2}\left(\mathbb{R}^{2}\right)$, and some constant $S_{2, q}>0$.

For $d=3$, one uses Theorem 8.3(i) in [3], which states (for $d=3$ ) $\|\nabla \phi\|_{2}^{2} \geqslant S_{3}\|\phi\|_{6}^{2}$ for some constant $S_{3}>0$, and Hölder's inequality, which implies $\|\phi\|_{4} \leqslant\|\phi\|_{6}^{3 / 4}\|\phi\|_{2}^{1 / 4}$.)

We estimate the Taylor expansion of $\Lambda_{\phi}(\sigma)$ at $\sigma_{0}=0$ up to second order for $\sigma \leqslant 0$, using (3.1) to express $\Lambda_{\phi}^{\prime}$ :

$$
\begin{gathered}
\Lambda_{\phi}(0)=0, \quad \Lambda_{\phi}^{\prime}(0)=\|\phi\|_{2}^{2}, \\
\Lambda_{\phi}^{\prime \prime}(\sigma)=\int_{\mathbb{R}^{d}} \phi^{4} \mathrm{e}^{\sigma \phi^{2}} \mathrm{~d} x \leqslant\|\phi\|_{4}^{4} .
\end{gathered}
$$

The Taylor expansion and the Sobolev inequality (3.8) yield for all $\sigma \leqslant 0$ :

$$
\Lambda_{\phi}(\sigma) \leqslant\|\phi\|_{2}^{2} \sigma+\frac{\|\phi\|_{4}^{4}}{2} \sigma^{2} \leqslant \sigma+\frac{c_{13}}{2} \sigma^{2} .
$$

Using Lemma 3.2 we get for $0<\mu<1$

$$
\Lambda^{*}(\mu) \geqslant \sup _{\sigma \leqslant 0}\left((\mu-1) \sigma-\frac{c_{13}}{2} \sigma^{2}\right)=\frac{1}{2 c_{13}}(1-\mu)^{2},
$$

and therefore with $c_{14}(d) \stackrel{\text { def }}{=} c_{12}\left(2 c_{13}\right)^{-2 / d}$, and $C_{1}(d) \stackrel{\text { def }}{=} c_{14}^{-d /(4-d)}(4 / d)^{-4 /(4-d)}(4 / d-$ 1) $>0$ :

$$
I(\beta) \geqslant \inf _{0<\mu<1}\left(c_{14}(1-\mu)^{4 / d}+\beta \mu\right) \geqslant \inf _{\mu<1}\left(c_{14}(1-\mu)^{4 / d}+\beta \mu\right)=\beta-C_{1} \beta^{4 /(4-d)} ;
$$

here the optimal point is $\mu=1-\left(d \beta /\left(4 c_{14}\right)\right)^{d /(4-d)}<1$. This proves the lower bound of Theorem 0.3 (the upper bound has been proven in Lemma 3.4 of [4]).

We provide now an alternative proof for the existence of a phase transition in dimensions $d \geqslant 4$, including a quantitative lower bound for the critical point. This proof does not make use of the Cwickel-Lieb-Rosenbljum theorem (see Theorem 9.3 of Simon [7], Theorem 2.1 of [4], resp.).

First we introduce the relevant constants:

$$
S_{d} \stackrel{\text { def }}{=} \frac{d(d-2)}{4}\left|\mathbb{S}^{d}\right|^{2 / d}=\frac{d(d-2)}{4} 2^{2 / d} \pi^{1+1 / d} \Gamma\left(\frac{1+d}{2}\right)^{-2 / d} ;
$$

here $\mathbb{S}^{d}$ denotes the unit sphere in $\mathbb{R}^{d+1} . S_{d}$ is the optimal constant in the Sobolev inequality (see Theorem 8.3 in [3])

$$
\|\nabla \phi\|_{2}^{2} \geqslant S_{d}\|\phi\|_{2 \gamma}^{2},
$$


with $\gamma \stackrel{\text { def }}{=} d /(d-2), d \geqslant 3, \phi \in D^{1}\left(\mathbb{R}^{n}\right) \supseteq H_{0}^{1,2}\left(\mathbb{R}^{n}\right)$ (for simplicity we skip here the formal definition of $D^{1}\left(\mathbb{R}^{n}\right)$ ). We set

$$
\beta_{0}(d) \stackrel{\text { def }}{=} c_{12} S_{d}\left(\frac{2}{d}\right)^{2 / d}=2^{2 / d-1} d^{-4 / d} S_{d}
$$

Proof of Theorem 0.4. - Let $d \geqslant 4$. Here we prove that for all $\beta \leqslant \beta_{0}$ we have $I(\beta) \geqslant \beta$. Using the concavity of $I$ (see Lemma 3.1) this implies that there exists $\beta_{c}(d) \geqslant \beta_{0}(d)>0$ such that $(0.11)-(0.12)$ is fulfilled. This also proves the lower bounds in $(0.13)-(0.14)$, whereas the upper bounds in $(0.13)-(0.14)$ are a consequence of Lemmas 3.4 and 3.5 below.

We choose $\gamma=d /(d-2)$, which fulfills $1<\gamma \leqslant 2$. We claim for all $\xi \leqslant 0$ :

$$
\mathrm{e}^{\xi}-1 \leqslant \xi+\frac{|\xi|^{\gamma}}{\gamma}
$$

To prove (3.17), we observe first that for all $s \leqslant 0$ :

$$
1-|s|^{\gamma-1} \leqslant \mathrm{e}^{s}
$$

this is obvious for $s \leqslant-1$ (in this case we have $1-|s|^{\gamma-1} \leqslant 0 \leqslant \mathrm{e}^{s}$ ). For $-1<s \leqslant 0$ we get (3.18) from $1-|s|^{\gamma-1} \leqslant 1-|s|=1+s \leqslant \mathrm{e}^{s}$, since $0<\gamma-1 \leqslant 1$. The bound (3.17) follows by integrating (3.18) over the interval $[\xi, 0]$.

Let $\sigma \leqslant 0, \phi \in \Phi,\|\nabla \phi\|_{2}=1$. We substitute $\sigma \phi^{2} \leqslant 0$ for $\xi$ in (3.17), this implies

$$
\Lambda_{\phi}(\sigma)=\int_{\mathbb{R}^{d}}\left(\mathrm{e}^{\sigma \phi^{2}}-1\right) \mathrm{d} x \leqslant\|\phi\|_{2}^{2} \sigma+\frac{\|\phi\|_{2 \gamma}^{2 \gamma}}{\gamma}|\sigma|^{\gamma} \leqslant \sigma+\frac{|\sigma|^{\gamma}}{\gamma S_{d}^{\gamma}},
$$

where in the last step we have used the Sobolev inequality (3.15), $\|\nabla \phi\|_{2}=1$, and $\|\phi\|_{2}=1$. Monotonicity of the Legendre transform (Lemma 3.2) yields for $\mu \in(0,1)$ :

$$
\Lambda_{\phi}^{*}(\mu) \geqslant \sup _{\sigma \leqslant 0}\left((\mu-1) \sigma-\frac{|\sigma|^{\gamma}}{\gamma S_{d}^{\gamma}}\right)=\frac{2}{d} S_{d}^{d / 2}(1-\mu)^{d / 2}
$$

the optimal point is $\sigma=-S_{d}^{\gamma /(\gamma-1)}(1-\mu)^{1 /(\gamma-1)}$, and we have used $d / 2=\gamma /(\gamma-1)$. We insert this result in (3.6) and get

$$
I(\beta) \geqslant \inf _{0<\mu<1}\left((1-\mu) \beta_{0}+\beta \mu\right)= \begin{cases}\beta & \text { for } 0<\beta \leqslant \beta_{0} \\ \beta_{0} & \text { for } \beta>\beta_{0}\end{cases}
$$

recall definition (3.16) of $\beta_{0}$. The proof of Theorem 0.4 is finished.

\subsection{Critical exponent in 4 dimensions (proof of Theorem 0.5)}

In this subsection we prove that for $d=4, \beta_{0}(4)$ is the critical $\beta$ (i.e. $\beta_{c}(4)=\beta_{0}(4)$ ) and that $I(\beta)$ is differentiable at $\beta_{c}(4)$. 
LEMmA 3.3. - Let $d=4$. There is a constant $C_{3}>0$ such that for all $\beta \geqslant \beta_{0}(4)$ :

$$
I(\beta) \geqslant \beta-C_{3}\left(\beta-\beta_{0}(4)\right)^{2} .
$$

Especially, $I$ is differentiable at the point $\beta=\beta_{0}(4)$.

Proof. - We use a similar technique as in the proof of Theorem 0.4. By convexity of the exponential function we know for all $y, s \in \mathbb{R}$ :

$$
\mathrm{e}^{s} \geqslant \mathrm{e}^{y}+\mathrm{e}^{y}(s-y)
$$

Let $\xi<0$. We integrate (3.23) over the interval $[\xi, 0]$ and obtain

$$
\mathrm{e}^{\xi}-1 \leqslant \mathrm{e}^{y}\left((1-y) \xi+2^{-1} \xi^{2}\right) .
$$

Let $\sigma \leqslant 0, \phi \in \Phi$ with $\|\nabla \phi\|_{2}=1$. We substitute $\xi=\sigma \phi^{2}$ in (3.24), integrate, and use Sobolev inequality (3.15) for $d=4, \gamma=2$ to get for all $y \in \mathbb{R}$ :

$$
\begin{aligned}
\Lambda_{\phi}(\sigma) & \leqslant \mathrm{e}^{y}\left((1-y) \sigma\|\phi\|_{2}^{2}+2^{-1} \sigma^{2}\|\phi\|_{4}^{4}\right) \\
& \leqslant \mathrm{e}^{y}\left((1-y) \sigma+2^{-1} S_{4}^{-2} \sigma^{2}\right) .
\end{aligned}
$$

We apply Lemma 3.2 to get for $\mu \in(0,1)$ :

$$
\begin{aligned}
\Lambda_{\phi}^{*}(\mu) & \geqslant \sup _{\sigma \leqslant 0}\left(\mu \sigma-\mathrm{e}^{y}\left((1-y) \sigma+\frac{\sigma^{2}}{2 S_{4}^{2}}\right)\right) \\
& = \begin{cases}\frac{S_{4}^{2}}{2}\left(\mathrm{e}^{-y / 2} \mu+\mathrm{e}^{y / 2}(y-1)\right)^{2} & \text { for } \mu \leqslant \mathrm{e}^{y}(1-y), \\
0 & \text { for } \mu>\mathrm{e}^{y}(1-y),\end{cases}
\end{aligned}
$$

here the optimal point is $\sigma=\left(\left(\mu \mathrm{e}^{-y}+y-1\right) \wedge 0\right) S_{4}^{2}$. An exact optimization over $y$ would lead to a transcendental equation for $y$; however, it is sufficient for our purposes to use an approximation to the optimal point: In (3.26) we choose

$$
y=\frac{2}{3}(\mu-1) \in\left[-\frac{2}{3}, 0\right] .
$$

We observe for this choice of $y$ that $\mu \leqslant(1+y)(1-y) \leqslant \mathrm{e}^{y}(1-y)$; i.e. the first case in (3.26) occurs. Consequently (3.26) tells us

$$
\begin{aligned}
\Lambda_{\phi}^{*}(\mu)^{1 / 2} & \geqslant \frac{S_{4}}{\sqrt{2}}\left(\frac{1}{3} \mathrm{e}^{(\mu-1) / 3}(5-2 \mu)-\mathrm{e}^{(1-\mu) / 3} \mu\right) \\
& =\frac{S_{4}}{\sqrt{2}}\left((1-\mu)+\sum_{n=2}^{\infty}\left[(-1)^{n}(1-2 n)+3 n-1\right] \frac{(1-\mu)^{n}}{3^{n} n !}\right) \\
& \geqslant \frac{S_{4}}{\sqrt{2}}\left((1-\mu)+\frac{(1-\mu)^{2}}{9}\right) ;
\end{aligned}
$$

one should note that all Taylor coefficients are positive. Next we introduce the constant $C_{3} \stackrel{\text { def }}{=} \frac{9}{4 \beta_{0}(4)}$. The inequality (3.28), the bound (3.3), and the definition (3.16) of $\beta_{0}(4)$ imply 


$$
\begin{aligned}
I(\beta) & \geqslant \inf _{\mu<1}\left(\beta_{0}(4)\left((1-\mu)+\frac{(1-\mu)^{2}}{9}\right)+\beta \mu\right) \\
& = \begin{cases}\beta-C_{3}\left(\beta-\beta_{0}(4)\right)^{2} & \text { for } \beta \geqslant \beta_{0}(4), \\
\beta & \text { for } 0<\beta<\beta_{0}(4) ;\end{cases}
\end{aligned}
$$

for $\beta>\beta_{0}$ (4) the optimal value is given by $\mu=1-2 C_{3}\left(\beta-\beta_{0}(4)\right)$, for $0<\beta \leqslant \beta_{0}(4)$ we choose $\mu \uparrow 1$. The differentiability of $I(\beta)$ at $\beta=\beta_{0}(4)$ is a consequence of the bound (3.29) and of the upper bound $I(\beta) \leqslant \beta$. This proves Lemma 3.3.

Lemma 3.4. - Let $d=4$. There are constants $C_{4}>0, C_{5}>0$, and $b_{2}>\beta_{0}(4)$ such that for all $\beta \in\left(\beta_{0}(4), b_{2}\right)$ the following estimate holds:

$$
I(\beta) \leqslant \beta-C_{4} \frac{\left(\beta-\beta_{0}(4)\right)^{2}}{\left|\log \left[C_{5}\left(\beta-\beta_{0}(4)\right)\right]\right|} .
$$

Proof. - Let $\phi \in \Phi$ with $\|\nabla \phi\|_{2}^{2}=1$. We derive an upper bound for $\Lambda_{\phi}^{\prime}(\sigma)$ : For $\sigma \leqslant 0$ :

$$
\Lambda_{\phi}^{\prime}(\sigma)=\int_{\mathbb{R}^{d}} \phi^{2} \mathrm{e}^{\sigma \phi^{2}} \mathrm{~d} x \leqslant\|\phi\|_{2}^{2}+\sigma\|\phi\|_{4}^{4}+\frac{\sigma^{2}}{2}\|\phi\|_{6}^{6}=1+\sigma\|\phi\|_{4}^{4}+\frac{\sigma^{2}}{2}\|\phi\|_{6}^{6} .
$$

The function $\Lambda_{\phi}^{\prime}:(-\infty, 0] \rightarrow(0,1]$ is monotonically increasing, and $-1+\sqrt{1-x} \geqslant$ $-x / 2-x^{2} / 2$ holds for all $x \leqslant 1$. Using these two facts and (3.31), we get for

$$
\mu \in \mathcal{I}_{\phi} \stackrel{\text { def }}{=}\left\{\mu \in(0,1): 1-\mu \leqslant \frac{\|\phi\|_{4}^{8}}{2\|\phi\|_{6}^{6}}\right\}
$$

the following lower bound, using (3.1) in the first step:

$$
\begin{aligned}
\Lambda_{\phi}^{* \prime}(\mu)=\Lambda_{\phi}^{\prime-1}(\mu) & =\sup \left\{\sigma \leqslant 0: \Lambda_{\phi}^{\prime}(\sigma) \leqslant \mu\right\} \\
& \geqslant \sup \left\{\sigma \leqslant 0: 1+\sigma\|\phi\|_{4}^{4}+\frac{\sigma^{2}}{2}\|\phi\|_{6}^{6} \leqslant \mu\right\} \\
& =\frac{\|\phi\|_{4}^{4}}{\|\phi\|_{6}^{6}}\left(-1+\sqrt{1-\frac{2\|\phi\|_{6}^{6}}{\|\phi\|_{4}^{8}}(1-\mu)}\right) \\
& \geqslant-\frac{1}{\|\phi\|_{4}^{4}}(1-\mu)-\frac{2\|\phi\|_{6}^{6}}{\|\phi\|_{4}^{12}}(1-\mu)^{2} .
\end{aligned}
$$

We integrate this estimate over an interval $(\mu, 1) \subseteq \mathcal{I}_{\phi}$; we obtain for $\mu \in \mathcal{I}_{\phi}$ :

$$
\begin{aligned}
\Lambda_{\phi}^{*}(\mu) & =\Lambda_{\phi}^{*}(1)-\int_{\mu}^{1} \Lambda_{\phi}^{* \prime}(\tilde{\mu}) \mathrm{d} \tilde{\mu} \leqslant \frac{1}{2\|\phi\|_{4}^{4}}(1-\mu)^{2}+\frac{2\|\phi\|_{6}^{6}}{3\|\phi\|_{4}^{12}}(1-\mu)^{3} \\
& \leqslant\left[\frac{1}{\sqrt{2}\|\phi\|_{4}^{2}}(1-\mu)+\frac{\sqrt{2}\|\phi\|_{6}^{6}}{3\|\phi\|_{4}^{10}}(1-\mu)^{2}\right]^{2} .
\end{aligned}
$$

Further we assume now that our test function $\phi$ satisfies the bound

$$
2\|\phi\|_{6}^{6} \geqslant\|\phi\|_{4}^{8}
$$


one should compare this with (3.32). We apply (3.34) and (3.3) for $\beta \in\left(0, \frac{5 c_{12}}{3 \sqrt{2}}\|\phi\|_{4}^{-2}\right)$ :

$$
\begin{aligned}
I(\beta) & \leqslant \inf _{\mu \in \mathcal{I}_{\phi}}\left(c_{12} \sqrt{\Lambda_{\phi}^{*}(\mu)}+\beta \mu\right) \\
& \leqslant \inf _{\mu \in \mathcal{I}_{\phi}}\left(\frac{c_{12}}{\sqrt{2}\|\phi\|_{4}^{2}}(1-\mu)+\frac{c_{12} \sqrt{2}\|\phi\|_{6}^{6}}{3\|\phi\|_{4}^{10}}(1-\mu)^{2}+\beta \mu\right) \\
& = \begin{cases}\beta & \text { for } \beta \leqslant \frac{c_{12}}{\sqrt{2}}\|\phi\|_{4}^{-2}, \\
\beta-\frac{3\|\phi\|_{4}^{10}}{4 \sqrt{2} c_{12}\|\phi\|_{6}^{6}}\left(\beta-\frac{c_{12}}{\sqrt{2}}\|\phi\|_{4}^{-2}\right)^{2} & \text { for } \frac{c_{12}}{\sqrt{2}}\|\phi\|_{4}^{-2}<\beta \leqslant \frac{5 c_{12}}{3 \sqrt{2}}\|\phi\|_{4}^{-2} .\end{cases}
\end{aligned}
$$

In view of this bound we need to maximize $\|\phi\|_{4}^{2}$ with the constraints $\phi \in \Phi,\|\nabla \phi\|_{2}^{2}=1$, and (3.35). Theorem 8.3 in [3] tells us: In the Sobolev inequality (3.15) $\left(\|\nabla \psi\|_{2}^{2} \geqslant\right.$ $S_{4}\|\psi\|_{4}^{2}, \psi \in D^{1}\left(\mathbb{R}^{4}\right)$ ) we have equality if we choose the function $\psi$ to be

$$
\psi(x)=\frac{1}{1+|x|^{2}}
$$

(and also for scaled and translated versions of it). However, this function $\psi$ is not compactly supported; we even have $\psi \notin L^{2}\left(\mathbb{R}^{4}\right)$. Therefore we introduce a truncated approximation $\psi_{R}$ of $\psi$ : Let $\chi: \mathbb{R}^{4} \rightarrow[0,1]$ denote any fixed smooth radially symmetric function which is compactly supported in $B_{2}(0)$ and equals 1 on $B_{1}(0)$. For $R>0$ we set

$$
\psi_{R}(x) \stackrel{\text { def }}{=} \frac{\chi(x / R)}{1+|x|^{2}} .
$$

We note that for some positive constants $\tilde{c}_{15}<c_{15}, c_{16}, \tilde{c}_{17}<c_{17}$, and $c_{18}$ the following bounds hold for large $R$ :

$$
\begin{aligned}
\tilde{c}_{15} \log R \leqslant\left\|\psi_{R}\right\|_{2}^{2} & \leqslant c_{15} \log R, \\
\|\psi\|_{4}^{4}-\frac{c_{16}}{R^{4}} & \leqslant\left\|\psi_{R}\right\|_{4}^{4} \leqslant\|\psi\|_{4}^{4}, \\
\tilde{c}_{17} & \leqslant\left\|\psi_{R}\right\|_{6}^{6} \leqslant c_{17},
\end{aligned}
$$

the last important inequality is

$$
\begin{aligned}
\left|\left\|\nabla \psi_{R}\right\|_{2}-\|\nabla \psi\|_{2}\right| & \leqslant\left\|\nabla\left(\psi_{R}-\psi\right)\right\|_{2}=\|\nabla(\psi(1-\chi(\cdot / R)))\|_{2} \\
& \leqslant\left\|1_{R \operatorname{supp}(1-\chi)} \nabla \psi\right\|_{2}+\|\nabla \chi\|_{\infty} R^{-1}\left\|1_{R \operatorname{supp}(\nabla \chi)} \psi\right\|_{2} \\
& \leqslant c_{18} R^{-1} ;
\end{aligned}
$$

where we have used polar coordinates to evaluate the integrals for the norms and we have inserted the following scaling behaviors: $|x|^{3}|\nabla \psi(x)|^{2} \leqslant \mathrm{O}\left(|x|^{-3}\right)$ and $|x|^{3}|\psi(x)|^{2} \leqslant$ $\mathrm{O}\left(|x|^{-1}\right)$ as $|x| \rightarrow \infty ; R \operatorname{supp}(\nabla \chi)$ is contained in an annulus centered at 0 with radii $R$ and $2 R$, and $R \operatorname{supp}(1-\chi)$ does not intersect $B_{R}(0)$. (3.40), (3.42), and equality in the Sobolev estimate (3.15) yield for some constant $c_{19}>0$ and large $R$ :

$$
S_{4} \leqslant \frac{\left\|\nabla \psi_{R}\right\|_{2}^{2}}{\left\|\psi_{R}\right\|_{4}^{2}} \leqslant \frac{\left(\|\nabla \psi\|_{2}+c_{18} R^{-1}\right)^{2}}{\left(\|\psi\|_{4}^{4}-c_{16} R^{-4}\right)^{1 / 2}} \leqslant S_{4}+\frac{c_{19}}{R} .
$$


In general $\psi_{R} \notin \Phi$; we still need to scale $\psi_{R}$ : We set

$$
\phi_{R}(x) \stackrel{\text { def }}{=} c \psi_{R}(x / r)
$$

where the scaling constants $c(R), r(R)$ are chosen such that $\left\|\phi_{R}\right\|_{2}^{2}=1=\left\|\nabla \phi_{R}\right\|_{2}^{2}$; to be specific, we have $r=\left\|\nabla \psi_{R}\right\|_{2} /\left\|\psi_{R}\right\|_{2}$ and $c=\left\|\psi_{R}\right\|_{2} /\left\|\nabla \psi_{R}\right\|_{2}^{2}$. Using further that

$$
\left\|\phi_{R}\right\|_{4}=c r\left\|\psi_{R}\right\|_{4} \quad \text { and } \quad\left\|\phi_{R}\right\|_{6}^{6}=c^{6} r^{4}\left\|\psi_{R}\right\|_{6}^{6},
$$

we obtain for large $R$ ( $c_{20}, c_{21}$ denote positive constants):

$$
\begin{aligned}
& \left\|\phi_{R}\right\|_{4}^{-2}=\frac{\left\|\nabla \psi_{R}\right\|_{2}^{2}}{\left\|\psi_{R}\right\|_{4}^{2}} \leqslant S_{4}+\frac{c_{19}}{R}, \\
& \frac{\left\|\phi_{R}\right\|_{4}^{10}}{\left\|\phi_{R}\right\|_{6}^{6}}=\frac{\left\|\psi_{R}\right\|_{4}^{10}}{\left\|\nabla \psi_{R}\right\|_{2}^{2}\left\|\psi_{R}\right\|_{2}^{2}\left\|\psi_{R}\right\|_{6}^{6}} \geqslant \frac{c_{20}}{\log R}, \\
& \frac{\left\|\phi_{R}\right\|_{4}^{8}}{\left\|\phi_{R}\right\|_{6}^{6}}=\frac{\left\|\psi_{R}\right\|_{4}^{8}}{\left\|\psi_{R}\right\|_{2}^{2}\left\|\psi_{R}\right\|_{6}^{6}} \leqslant \frac{c_{21}}{\log R} \stackrel{R \rightarrow \infty}{\longrightarrow} 0 ;
\end{aligned}
$$

especially assumption (3.35) is fulfilled for the test function $\phi_{R}$ for large $R$. Let $\beta>\beta_{0}(4)$. We set

$$
R=\frac{c_{12} c_{19} 2^{-1 / 2}}{\frac{1}{2}\left(\beta-\beta_{0}(4)\right)} .
$$

The bound (3.36) together with the estimates (3.46)-(3.48) and definition (3.16) of $\beta_{0}(4)$ yields for $R$ large enough, say $\beta \in\left(\beta_{0}(4), b_{2}\right)$ :

$$
\begin{aligned}
I(\beta) & \leqslant \beta-\frac{3 c_{20}}{4 \sqrt{2} c_{12}}(\log R)^{-1}\left(\beta-\beta_{0}(4)-\frac{c_{12} c_{19}}{\sqrt{2}} R^{-1}\right)^{2} \\
& =\beta-C_{4} \frac{\left(\beta-\beta_{0}(4)\right)^{2}}{\left|\log \left[C_{5}\left(\beta-\beta_{0}(4)\right)\right]\right|}
\end{aligned}
$$

for an appropriate choice of the positive constants $C_{4}, C_{5}$. This proves Lemma 3.4.

\subsection{Asymptotics in the large- $\beta$-region (proof of Theorem 0.2)}

The main tools to examine the large- $\beta$-behavior of $I(\beta)$ (especially for proving the limit (0.9)) are asymptotic upper and lower bounds for the ground state energy in a deterministic square-well potential. These bounds, well known to physicists at least in dimensions $d \leqslant 3$, are collected in Appendix B.

Let $r_{d}$ denote the radius of a $d$-dimensional ball of volume $d$. Here is an asymptotic upper bound for $I(\beta)$; we expect the exponent $-1 / 2$ of $\beta$ to be optimal:

LEMMA 3.5. - There are positive constants $b_{3}(d)$ and $c_{22}(d)$ such that for all $\beta \geqslant b_{3}$ :

$$
I(\beta) \leqslant c(d, 1)-\frac{c_{22}}{\sqrt{\beta}} .
$$


Proof. - Let $b_{3} \geqslant 2 c(d, 1)$ and $c_{22}$ be defined according to Lemma B.1 in Appendix B. Let $\beta \geqslant b_{3}$. By the upper bound in (B.1), there is a test function $\phi \in \Phi$ such that

$$
\frac{1}{2}\|\nabla \phi\|_{2}^{2}+\beta \mu \leqslant c(d, 1)-\frac{c_{22}}{\sqrt{\beta}}, \quad \text { where } \mu=\left\|1_{\mathbb{R}^{d} \backslash B_{r_{d}}(0)} \phi\right\|_{2}^{2} .
$$

This number $\mu$ cannot be 0 , i.e. $\phi$ cannot be supported in $\overline{B_{r_{d}}(0)}$, since this would imply $\|\nabla \phi\|_{2}^{2} / 2+\beta \mu \geqslant\|\nabla \phi\|_{2}^{2} / 2 \geqslant \lambda_{0}\left(B_{r_{d}}(0)\right)=c(d, 1)$, which contradicts (3.53). $\mu$ cannot equal 1 , since then $\|\nabla \phi\|_{2}^{2} / 2+\beta \mu \geqslant \beta \geqslant b_{3} \geqslant c(d, 1)$ which is a contradiction, too. It remains to examine $\mu \in(0,1)$ : We calculate, using the inequality $1+\xi-\mathrm{e}^{\xi} \leqslant 0$ :

$$
\begin{aligned}
\Lambda_{\phi}^{*}(\mu) & =\sup _{\sigma \leqslant 0} \mu \sigma-\int_{\mathbb{R}^{d}}\left(\mathrm{e}^{\sigma \phi^{2}}-1\right) \mathrm{d} x \\
& =\sup _{\sigma \leqslant 0} \int_{\mathbb{R}^{d} \backslash B_{r_{d}}(0)}\left(1+\sigma \phi^{2}-\mathrm{e}^{\sigma \phi^{2}}\right) \mathrm{d} x+\int_{B_{r_{d}}(0)}\left(1-\mathrm{e}^{\sigma \phi^{2}}\right) \mathrm{d} x \\
& \leqslant\left|B_{r_{d}}(0)\right|=d .
\end{aligned}
$$

Consequently the pair $\phi, \mu$ is an allowed test configuration in the definition (0.6) of $I$ : (0.6) and (3.53) together imply the bound (3.52). This proves Lemma 3.5.

Next we prove a lower bound for $I(\beta)$ in the large- $\beta$-region:

LEMMA 3.6. - There are constants $c_{23}(d)>0$ and $b_{4}(d)>1$ such that for all $\beta \geqslant b_{4}(d)$ the following lower bound holds:

$$
I(\beta) \geqslant c(d, 1)-c_{23} \beta^{-1 / 3} \log \beta .
$$

Proof. - We abbreviate "radially symmetric non-increasing" by "RSNI". Let $\phi^{\circ}$ denote the RSNI rearrangement of $\phi \in \Phi$ (see [3], Section 3.3). Then $\phi^{\circ} \in \Phi$, too, $\Lambda_{\phi}=\Lambda_{\phi^{\circ}}$, and therefore $\Lambda_{\phi}^{*}=\Lambda_{\phi^{\circ}}^{*}$. Lemma 7.17 in [3] shows $\left\|\nabla \phi^{\circ}\right\|_{2} \leqslant\|\nabla \phi\|_{2}$. We insert this in the definition (0.6) of $I(\beta)$ and scale $\phi$ (see (1.14)-(1.16)); this shows for every $m>0$ :

$$
I(\beta)=\inf \left\{\frac{m^{2 / d}}{2}\|\nabla \phi\|_{2}^{2}+\beta \mu: \phi \in \Phi \mathrm{RSNI}, \mu \in(0,1), \Lambda_{\phi}^{*}(\mu) \leqslant m d\right\} .
$$

The map $[0, \infty) \ni s \mapsto 1-(1+s) \mathrm{e}^{-s} \in[0,1)$ is bijective and monotonically increasing. Let $\beta>1$. We set $s=s(\beta) \stackrel{\text { def }}{=} \log \left(\beta^{1 / 3}\right)>0$ and $m=m(\beta) \stackrel{\text { def }}{=} 1-(1+s) \mathrm{e}^{-s} \stackrel{\beta \rightarrow \infty}{\longrightarrow} 1$. Further let $\phi \in \Phi$ RSNI, $\mu \in(0,1)$ with $\Lambda_{\phi}^{*}(\mu) \leqslant m d$, and $\sigma \stackrel{\text { def }}{=} \Lambda_{\phi}^{* \prime}(\mu) \leqslant 0$. We get (see (3.1))

$$
\begin{aligned}
m d & \geqslant \Lambda_{\phi}^{*}(\mu)=\sigma \mu-\Lambda_{\phi}(\sigma)=\int_{\mathbb{R}^{d}}\left[1-\left(1-\sigma \phi^{2}\right) \mathrm{e}^{\sigma \phi^{2}}\right] \mathrm{d} x \\
& \geqslant m\left|\left\{x \in \mathbb{R}^{d}:-\sigma \phi(x)^{2} \geqslant s\right\}\right|,
\end{aligned}
$$

and consequently $-\sigma \phi(x)^{2}<s$ for $|x|>r_{d}$, since $\left|B_{r_{d}}(0)\right|=d$, and since $\sqrt{|\sigma|} \phi$ is RSNI: the level set $\left\{x\right.$ : $\left.-\sigma \phi(x)^{2} \geqslant s\right\}$ is either empty or a ball centered at 0 . We 
multiply the inequality $1_{\mathbb{R}^{d} \backslash B_{r_{d}}(0)} \leqslant \mathrm{e}^{s} \mathrm{e}^{\sigma \phi^{2}}$ by $\phi^{2}$ and integrate; this yields the following inequality:

$$
\kappa \stackrel{\text { def }}{=}\left\|1_{\mathbb{R}^{d} \backslash B_{r_{d}}(0)} \phi\right\|_{2}^{2} \leqslant \mathrm{e}^{s} \int_{\mathbb{R}^{d}} \phi^{2} \mathrm{e}^{\sigma \phi^{2}} \mathrm{~d} x=\mathrm{e}^{s} \Lambda_{\phi}^{\prime}(\sigma)=\mathrm{e}^{s} \mu .
$$

The inequality $m^{2 / d} \geqslant 1-c_{24}(1-m)>0$ holds for some constant $c_{24}(d)>0$ and $m(\beta)<1$ sufficiently close to 1 , i.e. for large $\beta$. We combine the bound (3.58) and Corollary B.2 from Appendix B; we optimize over $\mu$; then we insert the lower bound for $m^{2 / d}$, use $m^{3 / d} \leqslant 1$, and abbreviate $c_{25} \stackrel{\text { def }}{=} 2 \cdot 3^{-3 / 2} c_{30}^{3 / 2}$, hence for large $\beta$ and some constant $c_{23}>0$ we get

$$
\begin{aligned}
\frac{m^{2 / d}}{2}\|\nabla \phi\|_{2}^{2}+\beta \mu & \geqslant m^{2 / d}\left(c(d, 1)-c_{30} \kappa^{1 / 3}\right)+\beta \mu \\
& \geqslant m^{2 / d}\left(c(d, 1)-c_{30} \mathrm{e}^{s / 3} \mu^{1 / 3}\right)+\beta \mu \\
& \geqslant m^{2 / d} c(d, 1)-c_{25} \mathrm{e}^{s / 2} m^{3 / d} \beta^{-1 / 2} \\
& \geqslant\left[1-c_{24}\left(1+\frac{1}{3} \log \beta\right) \beta^{-1 / 3}\right] c(d, 1)-c_{25} \beta^{-1 / 3} \\
& \geqslant c(d, 1)-c_{23} \beta^{-1 / 3} \log \beta .
\end{aligned}
$$

In view of (3.56) this proves Lemma 3.6.

\section{Appendix A. Upper bound of Theorem 0.6 and Corollary 0.7}

To prove the upper bound in Theorem 0.6 we simply evaluate (0.2) for a "good" test function.

Proof of the upper bound in Theorem 0.6. - In this proof we always assume that $\varphi(t)$ is a strictly positive function with $\varphi(t) \ll t$. Choose $t$ so large that $\varphi(t)<t / 2$. As test function we choose a function which is constant on $\mathcal{T}_{t-\varphi(t)}$ and with support contained in $\mathcal{T}_{t}$ : Let $\chi: \mathbb{R} \rightarrow[0,1]$ be any monotonically increasing $C^{\infty}$-function with support contained in $(0, \infty), \chi(x)=1$ for $x>1$, and $\chi^{\prime}(x) \leqslant 2$ for all $x \in \mathbb{R}$. We define

$$
\chi_{t}(x) \stackrel{\text { def }}{=} \chi\left(\frac{t+x}{\varphi(t)}\right) \cdot \chi\left(\frac{t-x}{\varphi(t)}\right) .
$$

The function $\chi_{t}$ is smooth, compactly supported in $(-t, t)$, constant 1 on $(-t+\varphi(t), t-$ $\varphi(t))$, and $\left|\chi_{t}^{\prime}(x)\right| \leqslant 2 / \varphi(t)$. Set $f_{t}(x) \stackrel{\text { def }}{=} \prod_{i=1}^{d} \chi_{t}\left(x_{i}\right)$ for $x=\left(x_{1}, \ldots, x_{d}\right) \in \mathbb{R}^{d}$, hence $f_{t}: \mathbb{R}^{d} \rightarrow[0,1]$ is smooth, compactly supported in $\mathcal{T}_{t}$, constant 1 on $\mathcal{T}_{t-\varphi(t)}$, and $\left|\nabla f_{t}\right| \leqslant$ $2 \sqrt{d} \varphi(t)^{-1}$. We have

$$
\left\|f_{t}\right\|_{2}^{2} \geqslant\left|\mathcal{T}_{t-\varphi(t)}\right| \quad \text { and } \quad\left\|\nabla f_{t}\right\|_{2}^{2} \leqslant\left\|\nabla f_{t}\right\|_{\infty}^{2}\left|\mathcal{T}_{t} \backslash \mathcal{T}_{t-\varphi(t)}\right| \leqslant 2 c_{26} t^{d-1} \varphi(t)^{-1},
$$

where $c_{26}(d) \stackrel{\text { def }}{=} 2^{d+1} d^{2}$. Our test function is defined by normalizing $f_{t}$ :

$$
\phi_{t} \stackrel{\text { def }}{=} \frac{f_{t}}{\left\|f_{t}\right\|_{2}},
$$


which satisfies $\left\|\phi_{t}\right\|_{2}=1$,

$$
\begin{aligned}
& \left\|\phi_{t}\right\|_{\infty} \leqslant\left\|f_{t}\right\|_{2}^{-1} \leqslant\left|\mathcal{T}_{t-\varphi(t)}\right|^{-1 / 2} \leqslant\left|\mathcal{T}_{t / 2}\right|^{-1 / 2} \\
& \left\|\nabla \phi_{t}\right\|_{2}^{2} \leqslant 2 c_{26} \frac{t^{d-1} \varphi(t)^{-1}}{\left|\mathcal{T}_{t / 2}\right|}=2 c_{26} t^{-1} \varphi(t)^{-1}
\end{aligned}
$$

Consequently

$$
\begin{aligned}
\lambda_{V_{\beta, t}}\left(\mathcal{T}_{t}\right) & \leqslant \frac{1}{2}\left\|\nabla \phi_{t}\right\|_{2}^{2}+\int_{\mathcal{T}_{t}} V_{\beta, t}(x) \phi_{t}^{2}(x) \mathrm{d} x \leqslant \frac{c_{26}}{t \varphi(t)}+\frac{\beta}{\varphi(t)^{2}}\|W\|_{1}\left\|\phi_{t}\right\|_{\infty}^{2} \omega\left(\mathcal{T}_{t}\right) \\
& \leqslant \frac{c_{26}}{t \varphi(t)}+\frac{\beta}{\varphi(t)^{2}} \frac{\omega\left(\mathcal{T}_{t}\right)}{\left|\mathcal{T}_{t-\varphi(t)}\right|}=\frac{c_{26}}{t \varphi(t)}+\left(1-\frac{\varphi(t)}{t}\right)^{-d} \frac{\beta}{\varphi(t)^{2}} \frac{\omega\left(\mathcal{T}_{t}\right)}{\left|\mathcal{T}_{t}\right|}
\end{aligned}
$$

By the ergodic theorem, $\mathbb{P}$-a.s. $\lim \sup _{t \rightarrow \infty}\left|\mathcal{T}_{t}\right|^{-1} \omega\left(\mathcal{T}_{t}\right) \leqslant 1$, and hence

$$
\mathbb{P} \text {-a.s. } \quad \limsup _{t \rightarrow \infty} \varphi(t)^{2} \lambda_{V_{\beta, t}}\left(\mathcal{T}_{t}\right) \leqslant \beta .
$$

The upper bound in Theorem 0.6 is proved.

Proof of Corollary 0.7. - By monotonicity we have for all $\beta, \beta_{1}>0$ and $\ell(t) \stackrel{\text { def }}{=}$ $(\log t)^{1 / d}$ :

$$
\begin{aligned}
& I\left(\beta_{1}\right) \stackrel{\text { P.a.s. }}{=} \liminf _{t \rightarrow \infty} \ell(t)^{2} \lambda_{V_{\beta_{1}, t}^{\ell}}\left(\mathcal{T}_{t}\right) \leqslant \liminf _{t \rightarrow \infty} \ell(t)^{2} \lambda_{V_{\beta, t}^{\varphi}}\left(\mathcal{T}_{t}\right) \\
& \leqslant \limsup _{t \rightarrow \infty} \ell(t)^{2} \lambda_{V_{\beta, t}^{\varphi}}\left(\mathcal{T}_{t}\right) \leqslant \limsup _{t \rightarrow \infty} \ell(t)^{2} \lambda_{0}\left(\mathcal{T}_{t} \backslash \operatorname{supp} V\right) .
\end{aligned}
$$

Now the claim follows from Theorem 0.2 and formula (4.4.38)-(4.4.40) in [9].

\section{Appendix B. Asymptotics of the ground state energy in a $d$-dimensional square well potential}

Recall, $r_{d}$ denotes the radius of a $d$-dimensional ball of volume $d$.

LEMMA B.1. - There are positive constants $b_{3}(d) \geqslant 2 c(d, 1)$ and $c_{22}(d)<\tilde{c}_{22}(d)$ such that for all $\beta \geqslant b_{3}$ the following holds:

$$
c(d, 1)-\frac{\tilde{c}_{22}}{\sqrt{\beta}}<\lambda_{\beta 1_{\mathbb{R}^{d} \backslash B_{r_{d}}(0)}}\left(\mathbb{R}^{d}\right)<c(d, 1)-\frac{c_{22}}{\sqrt{\beta}} .
$$

Proof. - By Theorem X.28 in [5] the Schrödinger operator $H_{\beta}=-\frac{1}{2} \Delta+\beta 1_{\mathbb{R}^{d} \backslash B_{r_{d}}(0)}$ is essentially self-adjoint on $C_{c}^{\infty}\left(\mathbb{R}^{d}\right)$. Theorem XIII.15 in [6] implies that the closure of $H_{\beta}$ has the essential spectrum $\sigma_{\text {ess }}\left(H_{\beta}\right)=\sigma_{\text {ess }}(-\Delta / 2+\beta)=[\beta, \infty)$. Therefore the infimum of the spectrum of $H_{\beta} \geqslant 0$ either equals $\beta$, or it is a discrete eigenvalue $E \in(0, \beta)$. ( $E=0$ is certainly impossible.) We show that the second case occurs at least for large values of $\beta$ : the potential $\beta 1_{\mathbb{R}^{d} \backslash B_{r_{d}}(0)}$ is radially symmetric; hence it suffices 
to look for the ground state eigenfunction among radially symmetric functions. We therefore search for weak solutions of the radial Schrödinger equation

$$
-\frac{1}{2}\left(\psi^{\prime \prime}(r)+\frac{d-1}{r} \psi^{\prime}(r)\right)+\beta 1_{\left\{r>r_{d}\right\}} \psi(r)=E \psi(r),
$$

that fulfill

$$
\int_{0}^{\infty}|\psi(r)|^{2} r^{d-1} \mathrm{~d} r<\infty .
$$

We solve piecewise the free radial Schrödinger equation explicitly in terms of (modified) Bessel functions: We get with the abbreviation $v=d / 2-1$ :

$$
\psi(r)= \begin{cases}(\sqrt{2 E} r)^{-v}\left(\alpha_{1} J_{v}(\sqrt{2 E} r)+\alpha_{3} Y_{v}(\sqrt{2 E} r)\right) & \text { for } r<r_{d}, \\ (\sqrt{2(\beta-E)} r)^{-v}\left(\alpha_{2} K_{v}(\sqrt{2(\beta-E)} r)+\alpha_{4} I_{v}(\sqrt{2(\beta-E)} r)\right) & \text { for } r>r_{d}\end{cases}
$$

with constants $\alpha_{1}, \alpha_{2}, \alpha_{3}, \alpha_{4}$. Regularity of the solution at the origin and condition (B.3) require $\alpha_{3}=0=\alpha_{4}$. Furthermore, $\psi$ and $\psi^{\prime}$ need to be continuous at $r=r_{d}$. We abbreviate $f(z) \stackrel{\text { def }}{=} z^{-v} J_{v}(z), g(z) \stackrel{\text { def }}{=} z^{-v} K_{v}(z), z_{1} \stackrel{\text { def }}{=} \sqrt{2 E} r_{d}$, and $z_{2} \stackrel{\text { def }}{=} \sqrt{2(\beta-E)} r_{d}$. For a given $E \in(0, \beta)$, we get a eigenfunction $\psi$ if and only if the following condition holds:

$$
\left|\begin{array}{cc}
f\left(z_{1}\right) & g\left(z_{2}\right) \\
z_{1} f^{\prime}\left(z_{1}\right) & z_{2} g^{\prime}\left(z_{2}\right)
\end{array}\right|=0
$$

Using the asymptotics

$$
\mathrm{e}^{z} \sqrt{\frac{2 z}{\pi}} K_{v}(z) \stackrel{z \rightarrow \infty}{\longrightarrow} 1, \quad \mathrm{e}^{z} \sqrt{\frac{2 z}{\pi}} K_{v}^{\prime}(z) \stackrel{z \rightarrow \infty}{\longrightarrow}-1
$$

(see [10], Section 7.23, and [1], formulas 9.7.2 and 9.7.4) we get

$$
-\frac{g(z)}{g^{\prime}(z)} \stackrel{z \rightarrow \infty}{\longrightarrow} 1
$$

Let $\xi$ denote the smallest positive number with $J_{v}(\xi)=0$. (Since the principal Dirichlet eigenfunction of $-\Delta / 2$ on the ball $B_{r_{d}}(0)$ is given by $x \mapsto f\left(\xi|x| / r_{d}\right)$ up to a normalizing constant, $\xi$ is related to Sznitman's constant by $c(d, 1)=$ $\left(\xi / r_{d}\right)^{2} / 2$.) The derivative $f^{\prime}(z)=-z^{-v} J_{v+1}(z)$ has no zero point $z$ in $(0, \xi]$ (see [10], Section 15.22). We calculate for $z \in(0, \xi]$, using an identity for Bessel functions from [10], Section 15.23:

$$
\frac{\mathrm{d}}{\mathrm{d} z}\left(-\frac{f(z)}{z f^{\prime}(z)}\right)=\left(z f^{\prime}(z)\right)^{-2}\left|\begin{array}{cc}
f(z) & z f^{\prime}(z) \\
\frac{\mathrm{d}}{\mathrm{d} z} f(z) & \frac{\mathrm{d}}{\mathrm{d} z}\left(z f^{\prime}(z)\right)
\end{array}\right|
$$




$$
\begin{aligned}
& =z^{-2 v-2} f^{\prime}(z)^{-2}\left|\begin{array}{cc}
J_{v}(z) & z J_{v}^{\prime}(z) \\
\frac{\mathrm{d}}{\mathrm{d} z} J_{v}(z) & \frac{\mathrm{d}}{\mathrm{d} z}\left(z J_{v}^{\prime}(z)\right)
\end{array}\right| \\
& =-2 z^{-2 v-3} f^{\prime}(z)^{-2} \int_{0}^{z} J_{v}^{2}(t) t \mathrm{~d} t<0 .
\end{aligned}
$$

Consequently $-f(z) /\left(z f^{\prime}(z)\right)$ is monotone decreasing on $(0, \xi]$ with the value zero at $\xi$ and a negative derivative at $\xi$. This shows that for some positive constants $c_{27}(d)<$ $c_{28}(d)$, and $c_{29}(d)<\xi$ the estimate $-f(z) /\left(z f^{\prime}(z)\right) \geqslant c_{27}(\xi-z)$ holds for all $z \in(0, \xi]$, and $-f(z) /\left(z f^{\prime}(z)\right) \leqslant c_{28}(\xi-z)$ holds for all $z \in\left[\xi-c_{29}, \xi\right]$. We choose a constant $\tilde{c}_{22}$ to be so large that $c_{27} r_{d}(2 c(d, 1))^{-1 / 2} \tilde{c}_{22}>2 r_{d}^{-1}$ holds, and we choose another constant $c_{22}>0$ so small that $2 c_{28} c(d, 1)^{-1 / 2} r_{d}^{2} c_{22}<1 / 2$ holds. Further we choose $b_{3}>0$ so large that the three conditions $2^{1 / 2} c(d, 1)^{-1 / 2} r_{d} c_{22} b_{3}^{-1 / 2} \leqslant c_{29}, b_{3} \geqslant 2 c(d, 1)$, and for all $z \geqslant b_{3}^{1 / 2} r_{d}:-g^{\prime}(z) / g(z) \in[1 / 2,2]$ hold true; see (B.7). Let $\beta \geqslant b_{3}$ and $E \in(0, c(d, 1))$. We first show that for $E \leqslant c(d, 1)-\tilde{c}_{22} \beta^{-1 / 2}$ Eq. (B.5) has no solution: Using $\beta \leqslant 2(\beta-E)$,

$$
\begin{aligned}
-\frac{f\left(z_{1}\right)}{z_{1} f^{\prime}\left(z_{1}\right)} & \geqslant c_{27}\left(\xi-z_{1}\right)=c_{27} r_{d}(\sqrt{2 c(d, 1)}-\sqrt{2 E}) \\
& \geqslant c_{27} r_{d}(2 c(d, 1))^{-1 / 2}(c(d, 1)-E) \geqslant c_{27} r_{d}(2 c(d, 1))^{-1 / 2} \tilde{c}_{22} \beta^{-1 / 2} \\
& >2 r_{d}^{-1} \beta^{-1 / 2} \geqslant \frac{2}{z_{2}} \geqslant-\frac{g\left(z_{2}\right)}{z_{2} g^{\prime}\left(z_{2}\right)} .
\end{aligned}
$$

This proves the lower bound in (B.1). To derive the upper bound in (B.1), we set $E=c(d, 1)-c_{22} \beta^{-1 / 2}$ and estimate:

$$
\begin{aligned}
\xi-z_{1} & =r_{d}(\sqrt{2 c(d, 1)}-\sqrt{2 E}) \\
& \leqslant 2(2 c(d, 1))^{-1 / 2} r_{d}(c(d, 1)-E)=2^{1 / 2} c(d, 1)^{-1 / 2} r_{d} c_{22} \beta^{-1 / 2} \\
& \leqslant c_{29},
\end{aligned}
$$

and therefore

$$
\begin{aligned}
-\frac{f\left(z_{1}\right)}{z_{1} f^{\prime}\left(z_{1}\right)} & \leqslant c_{28}\left(\xi-z_{1}\right) \leqslant c_{28} 2^{1 / 2} c(d, 1)^{-1 / 2} r_{d} c_{22} \beta^{-1 / 2} \\
& \leqslant 2 c_{28} c(d, 1)^{-1 / 2} r_{d}^{2} c_{22} z_{2}^{-1}<\frac{1}{2 z_{2}} \leqslant-\frac{g\left(z_{2}\right)}{z_{2} g^{\prime}\left(z_{2}\right)} .
\end{aligned}
$$

Comparing (B.9) with (B.11) we conclude that Eq. (B.5) has a solution $E \in(c(d, 1)-$ $\left.\tilde{c}_{22} \beta^{-1 / 2}, c(d, 1)-c_{22} \beta^{-1 / 2}\right)$. This finishes the proof of the upper bound in (B.1) and of Lemma B.1.

COROLLARY B.2. - There is a constant $c_{30}(d)>0$ such that for all functions $\phi \in \Phi$ :

$$
\frac{1}{2}\|\nabla \phi\|_{2}^{2} \geqslant c(d, 1)-c_{30}\left\|1_{\mathbb{R}^{d} \backslash B_{r_{d}}(0)} \phi\right\|_{2}^{2 / 3} .
$$


Proof. - We set $c_{30} \stackrel{\text { def }}{=}\left(3 \cdot 2^{-2 / 3} \tilde{c}_{22}^{2 / 3}\right) \vee\left(2^{1 / 3} c(d, 1) b_{3}^{1 / 2} \tilde{c}_{22}^{-1 / 3}\right)$, and we abbreviate $\kappa \stackrel{\text { def }}{=}\left\|1_{\mathbb{R}^{d} \backslash B_{r_{d}}(0)} \phi\right\|_{2}^{2}$. If $\kappa=0$, i.e. if $\phi$ is supported in $\overline{B_{r_{d}}(0)}$, then (B.12) is obvious, since $c(d, 1)$ is the principal Dirichlet eigenvalue of $-\Delta / 2$ over $B_{r_{d}}(0)$. So we may assume $\kappa>0$. Set $\beta=\left(2 \kappa / \tilde{c}_{22}\right)^{-2 / 3}$. There are two cases: If $\beta<b_{3}$, then (B.12) holds trivially, since in this case the right-hand side in (B.12) is negative: $c_{30} \kappa^{1 / 3} \geqslant c(d, 1)\left(b_{3} / \beta\right)^{1 / 2}>$ $c(d, 1)$. Else if $\beta \geqslant b_{3}$, then Lemma B.1 is applicable. The lower bound in (B.1) yields the claim (B.12):

$$
\frac{1}{2}\|\nabla \phi\|_{2}^{2} \geqslant c(d, 1)-\frac{\tilde{c}_{22}}{\sqrt{\beta}}-\beta \kappa=c(d, 1)-\frac{3 \tilde{c}_{22}^{2 / 3}}{2^{2 / 3}} \kappa^{1 / 3} \geqslant c(d, 1)-c_{30} \kappa^{1 / 3} .
$$

Corollary B.2 is proved.

\section{Acknowledgement}

We thank A.-S. Sznitman for drawing our attention to this topic and for providing interesting references. We are grateful to F. den Hollander for interesting discussions and for useful references.

\section{REFERENCES}

[1] M. Abramowitz, A. Stegun, Handbook of Mathematical Functions, Dover Publications, New York, 1972.

[2] M. van den Berg, E. Bolthausen, F. den Hollander, Moderate deviations for the volume of the Wiener sausage, Annals of Mathematics 153 (2) (2001) 355-406.

[3] E.H. Lieb, M. Loss, Analysis, in: Graduate Studies in Mathematics, Vol. 14, American Mathematical Society, RI, 1997.

[4] F. Merkl, M.V. Wüthrich, Phase transition of the principal Dirichlet eigenvalue in a scaled Poissonian potential, Probab. Theory Related Fields 119 (4) (2001) 475-507.

[5] M. Reed, B. Simon, Methods of Modern Mathematical Physics II: Fourier Analysis, Self Adjointness, Academic Press, San Diego, 1975.

[6] M. Reed, B. Simon, Methods of Modern Mathematical Physics IV: Analysis of Operators, Academic Press, San Diego, 1978.

[7] B. Simon, Functional Integration and Quantum Physics, Academic Press, New York, 1979.

[8] A.S. Sznitman, Shape theorem, Lyapounov exponents and large deviations for Brownian motion in a Poissonian potential, Comm. Pure. Appl. Math. 47 (12) (1994) 1655-1688.

[9] A.S. Sznitman, Brownian Motion, Obstacles and Random Media, Springer, Berlin, 1998.

[10] G.N. Watson, A Treatise on the Theory of Bessel Functions, in: Cambridge Mathematical Library, Cambridge University Press, Cambridge, 1996, reprinted. 